\title{
The Terwilliger Algebra Associated with a Set of Vertices in a Distance-Regular Graph*
}

\author{
HIROSHI SUZUKI \\ hsuzuki@icu.ac.jp \\ Department of Mathematics, International Christian University, Mitaka, Tokyo 181-8585, Japan
}

Received March 27, 2003; Revised October 1, 2004; Accepted October 19, 2004

\begin{abstract}
Let $\Gamma$ be a distance-regular graph of diameter $D$. Let $X$ denote the vertex set of $\Gamma$ and let $Y$ be a nonempty subset of $X$. We define an algebra $\mathcal{T}=\mathcal{T}(Y)$. This algebra is finite dimensional and semisimple. If $Y$ consists of a single vertex then $\mathcal{T}$ is the corresponding subconstituent algebra defined by $\mathrm{P}$. Terwilliger. We investigate the irreducible $\mathcal{T}$-modules. We define endpoints and thin condition on irreducible $\mathcal{T}$-modules as a generalization of the case when $Y$ consists of a single vertex. We determine when an irreducible module is thin. When the module is generated by the characteristic vector of $Y$, it is thin if and only if $Y$ is a completely regular code of $\Gamma$. By considering a suitable subset $Y$, every irreducible $\mathcal{T}(x)$-module of endpoint $i$ can be regarded as an irreducible $\mathcal{T}(Y)$-module of endpoint 0 .
\end{abstract}

Keywords: distance-regular graph, association scheme, subconstituent algebra, Terwilliger algebra, tight graph, completely regular code

\section{Introduction}

Let $\Gamma$ denote a distance-regular graph. In [45], P. Terwilliger introduced the subconstituent algebra (now often called the Terwilliger algebra) of $\Gamma$ with respect to one of its vertices (the "base vertex"). Since then, these algebras have been investigated in many papers [3, 4, 6-14, 16-19, 22, 23, 26, 43, 45-50]. In this paper we generalize some of this work by replacing the base vertex with a base subset of vertices. To describe our results more precisely we recall some facts.

Let $\Gamma$ denote a distance-regular graph with diameter $D$, vertex set $X$, and valency $k$. Let $\operatorname{Mat}_{X}(\boldsymbol{C})$ denote the complex algebra of matrices whose rows and columns are indexed by $X$ and whose entries are in the complex numbers $C$. Let $A_{i} \in \operatorname{Mat}_{X}(C)(0 \leq i \leq D)$ denote the $i$-th distance matrix of $\Gamma$. Then the adjacency matrix $A\left(=A_{1}\right)$ has $D+1$ distinct eigenvalues. Let $E_{i} \in \operatorname{Mat}_{X}(\boldsymbol{C})(0 \leq i \leq D)$ denote the primitive idempotents of $A$. The matrix subalgebra $\mathcal{M}$ generated by $A$ is called the Bose-Mesner algebra of $\Gamma$. It is well-known that $\mathcal{M}=\operatorname{Span}\left(A_{0}, A_{1}, \ldots, A_{D}\right)=\operatorname{Span}\left(E_{1}, E_{1}, \ldots, E_{D}\right)[1,2,24]$. Fix a base vertex $x \in X$. Let $E_{i}^{*}=E_{i}^{*}(x) \in \operatorname{Mat}_{X}(C)$ denote the projection onto the $i$-th subconstituent of $\Gamma$. The Terwilliger algebra of $\Gamma$ with respect to $x$ is the matrix subalgebra $\mathcal{T}=\mathcal{T}(x)$ generated by $A$ and $E_{0}^{*}, E_{1}^{*}, \ldots, E_{D}^{*}$.

*This research was partially supported by the Grant-in-Aid for Scientific Research (No. 12640039), Japan Society of the Promotion of Science. A part of the research was done when the author was visiting the Ohio State University. 
The algebra $\mathcal{T}$ is semisimple since it is generated by real symmetric matrices. Thus much of the structure of $\mathcal{T}$ is determined by its modules. Let $V=C^{X}$ denote the complex vector space consisting of column vectors whose entries are indexed by $X$. Let $W \subset V$ be an irreducible $\mathcal{T}$-module. Then $W$ is the direct sum of the nonzero subspaces among $E_{0}^{*} W, E_{1}^{*} W, \ldots, E_{D}^{*} W . W$ is said to be thin if $\operatorname{dim} E_{i}^{*} W \leq 1$ for every $i$. The index $\min \left\{i \mid 0 \leq i \leq D, E_{i}^{*} W \neq 0\right\}$ is called the endpoint of $W$. There is only one irreducible module of endpoint 0 and it is always thin. Recently Terwilliger investigated thin modules of endpoint 1 and obtained an inequality involving the local eigenvalues and showed that the equality holds if and only if every irreducible module of endpoint 1 is thin [48, 49]. Tight distance-regular graphs defined in [31] have lots of interesting properties, one of which is that every irreducible module of endpoint 1 is thin and of dimension $D-1$ [23]. Hence they are in the special class satisfying the equality mentioned above. For tight distance-regular graphs and related topics, see [27-30, 32, 33, 36-39, 44].

This paper is an attempt to generalize the results found in [23, 48, 49]. We first generalize the algebra $\mathcal{T}$ by replacing the base vertex by a base subset. Indeed let $Y$ be a nonempty subset of $X$. For $0 \leq i \leq D$ let $\Gamma_{i}(Y)$ denote the set of vertices in $X$ which are at distance $i$ from $Y$. Let $E_{i}^{*}=E_{i}^{*}(Y)$ denote the projection onto $\Gamma_{i}(Y)$. We let $\mathcal{T}=\mathcal{T}(Y)$ denote the subalgebra of $\operatorname{Mat}_{X}(\boldsymbol{C})$ generated by $A, E_{0}^{*}, E_{1}^{*}, \ldots, E_{D}^{*}$. Now we can define endpoints and the thin property similarly for irreducible $\mathcal{T}$-modules.

For a nonempty subset $Y$ of $X$ let $w(Y)$ denote the maximal distance of vertices of $Y$ in $\Gamma$, which is called the width of $Y$. Let $\boldsymbol{v} \in E_{0}^{*} V$ be a nonzero vector. Then the $\mathcal{T}$-module $\mathcal{T} v$ contains $\mathcal{M} v$. Moreover, if $\mathcal{T} v$ is a thin irreducible module, $\mathcal{T} v=\mathcal{M} v$.

We now state our main results, which will be proved in the body of the paper.

Our first result concerns the dimension of $\mathcal{M} \boldsymbol{v}$. Before we state our result we make a few comments. Observe that $\mathcal{M} v$ has a basis consisting of the nonzero vectors among $E_{0} v, E_{1} v, \ldots, E_{D} v$. Therefore,

$$
\operatorname{dim} \mathcal{M} \boldsymbol{v}=\left|\left\{i \mid E_{i} \boldsymbol{v} \neq \mathbf{0}, i \in\{0,1, \ldots, D\}\right\}\right|
$$

Theorem 1.1 Let $\Gamma=(X, R)$ be a distance-regular graph of diameter $D$, and let $Y$ denote a nonempty subset of $X$. Let $v$ be a nonzero vector in $E_{0}^{*}(Y) V$. Let $\mathcal{T}=\mathcal{T}(Y)$. Then the following hold.

(i) $\operatorname{dim} \mathcal{M} v \geq D-w(Y)+1$.

(ii) Suppose $\operatorname{dim} \mathcal{M} v=D-w(Y)+1$. Then $\mathcal{M} v$ is a thin irreducible $\mathcal{T}$-module.

In order to state our next result we make a few more comments. Under the assumption of Theorem 1.1, let $\operatorname{dim} \mathcal{M} v=r+1$. Since $A_{i}$ is expressed as a polynomial in $A$ of degree $i, \mathcal{M} v$ has another basis $A_{i} v(0 \leq i \leq r)$. Applying Gram-Schmidt to this basis, we find $\mathcal{M} \boldsymbol{v}$ has an orthogonal basis $g_{i}(A) \boldsymbol{v}(0 \leq i \leq r)$, where $g_{i}(t) \in \boldsymbol{R}[t]$ is a polynomial with degree exactly $i$ with leading coefficient $\left(c_{1} c_{2} \ldots c_{i}\right)^{-1}$. We compute $\left\|g_{i}(A) v\right\|^{2}$ in terms of the intersection numbers and the scalars

$$
\frac{{ }^{t} \boldsymbol{v} A_{i} \overline{\boldsymbol{v}}}{{ }^{t} \boldsymbol{v} \overline{\boldsymbol{v}}}, \quad \text { for } i \in\{0,1, \ldots, w(Y)\}
$$


We show that for $0 \leq j \leq r$,

$$
\left\|E_{i}^{*} A_{i} v\right\|^{2} \leq\left\|g_{i}(A) v\right\|^{2}
$$

Concerning the case of equality we have the following.

Theorem 1.2 Let $\Gamma=(X, R)$ be a distance-regular graph of diameter $D$, and let $Y$ denote a nonempty subset of $X$. Let $v$ be a nonzero vector in $E_{0}^{*}(Y) V$ and define $r=\operatorname{dim} \mathcal{M}-1$. Let $\mathcal{T}=\mathcal{T}(Y)$. Then the following are equivalent.

(i) $\mathcal{T} v$ is a thin irreducible $\mathcal{T}$-module.

(ii) Equality holds in (1) for $i \in\{0,1, \ldots, r\}$.

(iii) Equality holds in (1) for $i=r$.

When $v$ is the characteristic vector of $Y$, we show $\mathcal{M} v$ is an irreducible $\mathcal{T}$-module if and only if $Y$ is a completely regular code. See Proposition 7.2. Hence our attempt is to provide a new tool to study codes by considering not only the module generated by the characteristic vector of a code but also those generated by nonzero vectors whose supports lie in the code. For algebraic characterizations of completely regular codes, see [2, 20, 21, 24, 25, 34, 35, 41].

Let $W$ be an irreducible $\mathcal{T}(x)$-module of endpoint $i$. Then the same subspace $W$ becomes an irreducible $\mathcal{T}(Y)$-module of endpoint 0 by setting $Y=\Gamma_{i}(x)$. Hence by considering a suitable subset $Y$, every irreducible $\mathcal{T}(x)$-module can be regarded as an irreducible $\mathcal{T}(Y)$ module of endpoint 0 . Thus it is advantageous to choose a base subset rather than a base vertex. In fact the results in [23,48,49] are those on $\mathcal{T}\left(\Gamma_{1}(x)\right)$-modules. Since $w\left(\Gamma_{1}(x)\right)=2$, our results are generalization to the case with arbitrary width. We believe that our results set a foundation to investigate $\mathcal{T}(x)$-modules of endpoint larger than one as well.

The paper is organized as follows. The next two sections are for preliminaries. Section 2 is for orthogonal polynomials and Section 3 is for distance-regular graphs. The readers who are familiar with distance-regular graphs may skip these two sections and come back when quoted. In Section 4, we define Terwilliger algebras and their modules with respect to a base subset, and prove some basic results. Section 5 deals with the properties of change of base subsets. In Section 6, we discuss the structures of thin modules. In Section 7, we study the primary module. In Section 8 , we collect basic properties of local eigenvalues. In Sections 9 and 10, we prove our main theorems. In Section 11, we give description of the case when the width $w(Y)$ of $Y$ is at most 2. This contains the case when $Y=\Gamma_{1}(x)$, which was studied in $[23,48,49]$.

\section{Preliminaries: Orthogonal polynomials}

In this section we will review some of the basic properties of orthogonal polynomials. Orthogonal polynomials frequently arise in connection with distance-regular graphs [1, $2,24]$, and they play a fundamental role in our work. Thus we take a moment to recall some basic properties of orthogonal polynomials (most of this material is found in Szegö's book [40], see also [5, 15, 24]). To make these results more directly applicable to our 
situation, we specialize them to the orthogonal polynomials with associated weight functions having only a finite number of points of increase.

Definition 2.1 Let $X=\left\{x_{0}, x_{1}, \ldots, x_{N}\right\}$ be the set of $N+1$ distinct real numbers. Let $w$ be a real-valued function on the set $X$ such that $w(x)>0$ for all $x \in X$. Let $\boldsymbol{R}[t]$ be the set of polynomials in $t$ with real coefficients. For $f(t), g(t) \in \boldsymbol{R}[t]$, let

$$
\langle f(t), g(t)\rangle_{w}=\sum_{x \in X} f(x) g(x) w(x)=\sum_{i=0}^{N} f\left(x_{i}\right) g\left(x_{i}\right) w\left(x_{i}\right) .
$$

Then $\langle,\rangle_{w}$ defines an inner product on $\boldsymbol{R}[t]$. A system of polynomials $\left\{p_{0}(t), p_{1}(t), \ldots\right.$, $\left.p_{n}(t)\right\} \subset \boldsymbol{R}[t]$ is called a system of orthogonal polynomials on $X$ associated with the weight function $w$, if they are orthogonal with respect to the inner product $\langle,\rangle_{w}$ and that $\operatorname{deg}\left(p_{i}(t)\right)=i$. A system of orthogonal polynomials $\left\{p_{0}(t), p_{1}(t), \ldots, p_{n}(t)\right\} \subset \boldsymbol{R}[t]$ is called orthonormal if $\left\langle p_{i}(t), p_{i}(t)\right\rangle_{w}=1$ for every $i \in\{0,1, \ldots, n\}$.

If $\left\{p_{0}(t), p_{1}(t), \ldots, p_{n}(t)\right\} \subset \boldsymbol{R}[t]$ is a system of orthogonal polynomials, then every polynomial $p(t) \in \boldsymbol{R}[t]$ of degree at most $m$ with $m \leq n$ can be written as an $\boldsymbol{R}$-linear combination of $p_{0}(t), p_{1}(t), \ldots, p_{m}(t)$ and $\left\langle p(t), p_{i}(t)\right\rangle_{w}=0$ for every $i \in\{m+1, \ldots, n\}$.

Proposition 2.1 ([40]) Let $X=\left\{x_{0}, x_{1}, \ldots, x_{N}\right\}$ be the set of $N+1$ distinct real numbers. Let $w$ be a real-valued function on the set $X$ such that $w(x)>0$ for all $x \in X$. Let $f_{0}(t)=1$, $f_{i}(t) \in \boldsymbol{R}[t]$ such that $\operatorname{deg} f_{i}(t)=i$ for $i \in\{1, \ldots, N\}$.

(i) Let $c_{i, j}=\left\langle f_{i}(t), f_{j}(t)\right\rangle_{w}$ for every $i, j \in\{0,1, \ldots, N\}$. Then for every $n \in\{0,1, \ldots$, $N\}$ the value of the following determinant is positive.

$$
D_{n}=\left|\begin{array}{ccccc}
c_{0,0} & c_{0,1} & c_{0,2} & \ldots & c_{0, n} \\
c_{1,0} & c_{1,1} & c_{1,2} & \ldots & c_{1, n} \\
\ldots \ldots \ldots \ldots \ldots \ldots \ldots \ldots \ldots \ldots \ldots & \ldots \ldots \ldots \\
c_{n-1,0} & c_{n-1,1} & c_{n-1,2} & \cdots & c_{n-1, n} \\
c_{n, 0} & c_{n, 1} & c_{n, 2} & \ldots & c_{n, n}
\end{array}\right| .
$$

(ii) Let $\left\{p_{0}(t), p_{1}(t), \ldots, p_{N}(t)\right\} \subset \boldsymbol{R}[t]$ be a system of polynomials defined as follows.

$$
p_{0}(t)=1 \text {, }
$$

and for $n \in\{1,2, \ldots, N\}$

$$
p_{n}(t)=\left(D_{n-1}\right)^{-1}\left|\begin{array}{ccccc}
c_{0,0} & c_{0,1} & c_{0,2} & \ldots & c_{0, n} \\
c_{1,0} & c_{1,1} & c_{1,2} & \ldots & c_{1, n} \\
\ldots \ldots \ldots & \ldots \ldots \ldots \ldots \ldots \ldots \ldots \ldots \ldots \\
c_{n-1,0} & c_{n-1,1} & c_{n-1,2} & \cdots & c_{n-1, n} \\
f_{0}(t) & f_{1}(t) & f_{2}(t) & \cdots & f_{n}(t)
\end{array}\right| .
$$


Then for each $n \in\{0,1, \ldots, N\}, p_{n}(t)$ is an $\boldsymbol{R}$-linear combination of $f_{0}(t), f_{1}(t), \ldots$ $f_{n}(t)$ and the coefficient of $f_{n}(t)$ is 1 . Moreover, $\left\{p_{0}(t), p_{1}(t), \ldots, p_{N}(t)\right\}$ is a system of orthogonal polynomials satisfying the following.

$$
\left\langle p_{i}(t), p_{j}(t)\right\rangle_{w}=\delta_{i, j} D_{i} .
$$

(iii) Suppose $\left\{q_{0}(t), q_{1}(t), \ldots, q_{N}(t)\right\}$ is a system of orthogonal polynomials on $X$ associated with a weight function $w$. Assume the following.

(a) The leading coefficient of $q_{i}(t)$ is the same as that of $f_{i}(t)$ for every $i \in\{0,1, \ldots$, $N\}$.

(b) $\left\langle q_{i}(t), q_{j}(t)\right\rangle_{w}=\delta_{i, j} D_{i}$ for every $i, j \in\{0,1, \ldots, N\}$, where $D_{i}$ is the determinant defined in (i).

Then $q_{i}(t)=p_{i}(t)$ for each $i \in\{0,1, \ldots, N\}$, where $p_{i}(t)$ is a polynomial defined in (ii).

Remark. By Lemma 2.1, if $w$ is a weight function on the set $X$ of size $N+1$, then there exists a system of $N+1$ orthogonal polynomials $\left\{p_{0}(t), p_{1}(t), \ldots, p_{N}(t)\right\}$ associated with $w$, but no larger system.

Lemma $2.2([24,40]) \quad$ Let $\left\{f_{0}(t), f_{1}(t), \ldots, f_{N}(t)\right\} \subset \boldsymbol{R}[t]$ be a system of orthogonal polynomials on the set $X$ of cardinality $N+1$ associated with the weight function $w$. Set $f_{N+1}(t)=\prod_{x \in X}(t-x)$. Then for each $n \in\{0,1, \ldots, N\}$, there exist real numbers $\beta_{n-1}, \alpha_{n}$ and $\gamma_{n+1}$ satisfying the following relations.

$$
t f_{n}(t)=\beta_{n-1} f_{n-1}(t)+\alpha_{n} f_{n}(t)+\gamma_{n+1} f_{n+1}(t),
$$

and that

$$
\frac{\left\langle f_{n+1}(t), f_{n+1}(t)\right\rangle_{w}}{\left\langle f_{n}(t), f_{n}(t)\right\rangle_{w}}=\frac{\beta_{n}}{\gamma_{n+1}}, \quad \text { for every } n \in\{0,1, \ldots, N-1\} .
$$

In particular, $\beta_{n} \gamma_{n+1}>0$ for every $n \in\{0,1, \ldots, N-1\}$.

Lemma 2.3 Let $\left\{p_{0}(t), p_{1}(t), \ldots, p_{N}(t)\right\} \subset \boldsymbol{R}[t]$ be a system of orthogonal polynomials on $X$ associated with the weight function $w$. Let $\rho(t) \in \boldsymbol{R}[t]$ such that $\rho(x) \geq 0$ for every $x \in X$. Suppose

$$
\rho(t)=c\left(t-y_{1}\right)\left(t-y_{2}\right) \cdots\left(t-y_{\ell}\right) \quad(0 \neq c \in \boldsymbol{R}),
$$

where $y_{1}, y_{2}, \ldots, y_{\ell}$ are distinct elements of $X$. Let $D_{\rho, n}$ be a determinant of size $\ell \times \ell$ defined by the following.

$$
D_{\rho, n}=\left|\begin{array}{cccc}
p_{n}\left(y_{1}\right) & p_{n+1}\left(y_{1}\right) & \ldots & p_{n+\ell-1}\left(y_{1}\right) \\
p_{n}\left(y_{2}\right) & p_{n+1}\left(y_{2}\right) & \ldots & p_{n+\ell-1}\left(y_{2}\right) \\
\ldots \ldots \ldots \ldots \ldots \ldots \ldots \ldots \ldots \ldots \ldots \ldots & \ldots \ldots \ldots \ldots \ldots \\
p_{n}\left(y_{\ell}\right) & p_{n+1}\left(y_{\ell}\right) & \ldots & p_{n+\ell-1}\left(y_{\ell}\right)
\end{array}\right| .
$$

Then $D_{\rho, n} \neq 0$ for every $n \in\{0,1, \ldots, N-\ell+1\}$. 
Proof: Suppose $D_{\rho, n}=0$. Then there is a nontrivial linear combination of columns of the determinant. Let $\boldsymbol{u}_{i}$ be the $(i+1)$-st column of the determinant (4) as follows:

$$
{ }^{t}\left(p_{n+i}\left(y_{1}\right), p_{n+i}\left(y_{2}\right), \ldots, p_{n+i}\left(y_{\ell}\right)\right)
$$

for $i=0,1, \ldots, \ell-1$. Suppose $\lambda_{0}, \lambda_{1}, \ldots, \lambda_{\ell-1}$ are certain constants of real numbers, not all zero, such that

$$
\lambda_{0} \boldsymbol{u}_{0}+\lambda_{1} \boldsymbol{u}_{1}+\cdots+\lambda_{\ell-1} \boldsymbol{u}_{\ell-1}=\mathbf{0} .
$$

and set

$$
p(t)=\lambda_{0} p_{n}(t)+\lambda_{1} p_{n+1}(t)+\lambda_{2} p_{n+2}(t)+\cdots+\lambda_{\ell-1} p_{n+\ell-1}(t) \in \boldsymbol{R}[t] .
$$

Then the degree of $p(t)$ is at most $n+\ell-1$. By (5), we have

$$
p\left(y_{i}\right)=0 \quad \text { for all } i \in\{1,2, \ldots, \ell\} .
$$

Thus $\rho(t)$ divides $p(t)$, and there is a nonzero polynomial $q(t) \in \boldsymbol{R}[t]$ of degree at most $n-1$ such that $p(t)=\rho(t) q(t)$. Since $p(t)$ is an $\boldsymbol{R}$-linear combination of $p_{n}(t), p_{n+1}(t), \ldots$, $p_{n+\ell-1}(t), p(t)$ is orthogonal to any polynomial in $\boldsymbol{R}[t]$ of degree at most $n-1$. In particular,

$$
\begin{aligned}
0 & =\langle p(t), q(t)\rangle_{w}=\langle\rho(t) q(t), q(t)\rangle_{w}=\sum_{x \in X} \rho(x) q(x) q(x) w(x) \\
& =\sum_{x \in X}|q(x)|^{2} \rho(x) w(x) .
\end{aligned}
$$

Since $\rho(x) \geq 0$ and $w(x)>0$ for all $x \in X$, we have that

$$
q(x)=0 \quad \text { for every } x \in X \backslash\left\{y_{1}, y_{2}, \ldots, y_{\ell}\right\} .
$$

Hence if $N+1-\ell>n-1$, we have $q(t)=0$ as a polynomial, which is a contradiction. Thus $D_{\rho, n} \neq 0$ if $n \leq N+1-\ell$ as desired.

\section{Preliminaries: Distance-regular graphs}

In this section we recall some facts about distance-regular graphs. For the general theory of distance-regular graphs, we refer the reader to $[1,2,24]$. We shall follow the notation of [1] for the most part.

Let $X$ denote a nonempty finite set. Let $\operatorname{Mat}_{X}(\boldsymbol{C})$ denote the complex algebra consisting of all matrices whose rows and columns are indexed by $X$ with complex entries. Let $V=C^{X}$ denote the vector space over the complex number field consisting of column vectors whose coordinates are indexed by $X$ with complex entries. We observe $\operatorname{Mat}_{X}(\boldsymbol{C})$ acts on $V$ by left 
multiplication. We endow $V$ with the Hermitean inner product $\langle$,$\rangle defined by$

$$
\langle u, v\rangle={ }^{t} u \bar{v}(u, v \in V),
$$

where ${ }^{t} \boldsymbol{u}$ denotes the transpose of $\boldsymbol{u}$, and $\overline{\boldsymbol{v}}$ denotes the complex conjugate of $\boldsymbol{v}$. We abbreviate $\|\boldsymbol{u}\|^{2}=\langle\boldsymbol{u}, \boldsymbol{u}\rangle$ for all $\boldsymbol{u} \in V$. For all $y \in X$, let $\hat{y}$ denote the element of $V$ with a 1 in the $y$-coordinate and 0 in all other coordinates.

Let $\Gamma=(X, R)$ denote a finite, undirected, connected graph, without loops or multiple edges, with vertex set $X$ and edge set $R$. For $x, y \in X$, let $\partial(x, y)$ denote the distance between $x$ and $y$, that is the length of a shortest path connecting $x$ and $y$. The diameter $D$ is the maximal distance between vertices. The graph $\Gamma$ is said to be distance-regular whenever for all integers $h, i, j \in\{0,1, \ldots, D\}$ and for all vertices $x, y \in X$ with $\partial(x, y)=h$, the number

$$
p_{i, j}^{h}=|\{z \in X \mid \partial(x, z)=i, \partial(z, y)=j\}|
$$

is independent of $x$ and $y$. These integers are called the intersection numbers of $\Gamma$. We abbreviate $c_{i}=p_{i-1,1}^{i}(1 \leq i \leq D), a_{i}=p_{i, 1}^{i}(0 \leq i \leq D)$, and $b_{i}=p_{i+1,1}^{i}(0 \leq i \leq$ $D-1)$. For notational convenience, we define $c_{0}=0$ and $b_{D}=0$.

For the rest of this paper we assume $\Gamma$ is distance-regular with diameter $D$. By (6) and the triangular inequality,

$$
p_{i, j}^{h}=0 \quad \text { if } h+i<j, i+j<h, \text { or } j+h<i .
$$

For a vertex $x \in X$ and an integer $i \in\{0,1, \ldots, D\}$, let

$$
\Gamma_{i}(x)=\{y \in X \mid \partial(x, y)=i\} \quad \text { and } \quad k_{i}=\left|\Gamma_{i}(x)\right| .
$$

Then $k_{i}=p_{i, i}^{0}$ and the induced graph on $\Gamma_{i}(x)$ is an $a_{i}$-regular graph of size $k_{i}$, and $k=k_{1}=c_{i}+a_{i}+b_{i}$ for every $i \in\{0,1, \ldots, D\}$. It is known that each $p_{i, j}^{h}$ can be written using $c_{i}$ 's, $a_{i}$ 's and $b_{i}$ 's. We refer to $\left\{p_{i, j}^{h} \mid h, i, j \in\{0,1, \ldots, D\}\right\}$ as the set of parameters of $\Gamma$.

For $i \in\{0,1, \ldots, D\}$ let $A_{i}$ denote the matrix in $\operatorname{Mat}_{X}(\boldsymbol{C})$ whose $(x, y)$-entry is defined by

$$
\left(A_{i}\right)_{x, y}= \begin{cases}1 & \text { if } \partial(x, y)=i \\ 0 & \text { otherwise }\end{cases}
$$

The matrix $A_{i}$ is called the $i$-th distance matrix of $\Gamma$. For $i, j \in\{0,1, \ldots, D\}$ we have

$$
A_{i} A_{j}=\sum_{h=0}^{D} p_{i, j}^{h} A_{h}
$$


In particular, by (7) we have for $i \in\{1, \ldots, D\}$

$$
A_{i} A_{1}=b_{i-1} A_{i-1}+a_{i} A_{i}+c_{i+1} A_{i+1}
$$

by setting $A_{D+1}=0$ and $c_{D+1}=1$. Observe that by (8), the linear span $\mathcal{M}=\operatorname{Span}\left(A_{0}\right.$, $\left.A_{1}, \ldots, A_{D}\right)$ is closed under multiplication and it is algebraically generated by $A=A_{1} . \mathcal{M}$ is called the Bose-Mesner algebra of $\Gamma$. Since $\mathcal{M}$ is commutative and generated by real symmetric matrices, it has a basis consisting of primitive idempotents. Let $E_{0}, E_{1}, E_{2}, \ldots, E_{D}$ be the primitive idempotents. We write

$$
A_{i}=\sum_{j=0}^{D} p_{i}(j) E_{j} \quad \text { for all } i \in\{0,1, \ldots, D\},
$$

and

$$
E_{i}=\frac{1}{|X|} \sum_{j=0}^{D} q_{i}(j) A_{j} \quad \text { for all } i \in\{0,1, \ldots, D\}
$$

Set $m_{i}=q_{i}(0)$ and $\theta_{i}=p_{1}(i)$. Then $\theta_{0}, \theta_{1}, \ldots, \theta_{D}$ are the distinct eigenvalues of $A=A_{1}$, and $m_{i}$ is the multiplicity of $\theta_{i}$ in $A$. We order $E_{0}, E_{1}, \ldots, E_{D}$ so that

$$
\theta_{0}>\theta_{1}>\cdots>\theta_{D}
$$

Since $\Gamma$ is a connected $k$-regular graph, its adjacency matrix has largest eigenvalue $k$ with multiplicity 1 . Hence $E_{0}=\frac{1}{|X|} J$ in this ordering, where $J \in \operatorname{Mat}_{X}(\boldsymbol{C})$ is the all 1's matrix. We use the following well-known formulas. For all $i, j \in\{0,1, \ldots, D\}$,

$$
\begin{aligned}
\frac{p_{i}(j)}{k_{i}} & =\frac{q_{j}(i)}{m_{j}}, \\
q_{j}(1) & =\frac{m_{j} \theta_{j}}{k}, \\
q_{j}(2) & =\frac{m_{j}\left(\theta_{j}^{2}-a_{1} \theta_{j}-k\right)}{k b_{1}} .
\end{aligned}
$$

\section{Lemma 3.1 ( [1, 23]) The following hold.}

(i) Let $v_{0}(t), v_{1}(t), \ldots, v_{D}(t), v_{D+1}(t)$ denote polynomials in $\boldsymbol{R}[t]$ satisfying $v_{0}(t)=1$ and for $i \in\{0,1, \ldots, D\}$,

$$
t v_{i}(t)=b_{i-1} v_{i-1}(t)+a_{i} v_{i}(t)+c_{i+1} v_{i+1}(t),
$$

where $b_{-1}=0, c_{D+1}=1$ and $v_{-1}(t)=0$. Then for each integer $i \in\{0,1, \ldots, D+1\}$, the polynomial $v_{i}(t)$ has degree $i$, the leading coefficient $\left(c_{1} c_{2} \cdots c_{i}\right)^{-1}$, and $v_{i}\left(A_{1}\right)=$ 
$A_{i}$ with $A_{D+1}=$ O. Moreover,

$$
c_{1} c_{2} \ldots c_{D} v_{D+1}(t)=\left(t-\theta_{0}\right)\left(t-\theta_{1}\right) \cdots\left(t-\theta_{D}\right)
$$

and for $i, j \in\{0,1, \ldots, D\}$,

$$
\sum_{h=0}^{D} v_{i}\left(\theta_{h}\right) v_{j}\left(\theta_{h}\right) m_{h}=\delta_{i, j} k_{i}|X| .
$$

(ii) Let $p_{0}(t), p_{1}(t), \ldots, p_{D}(t)$ denote polynomials in $\boldsymbol{R}[t]$ satisfying $p_{0}(t)=1$ and for $i \in\{0,1, \ldots, D-1\}$,

$$
t p_{i}(t)=b_{i} p_{i-1}(t)+\left(a_{i}-c_{i+1}+c_{i}\right) p_{i}(t)+c_{i+1} p_{i+1}(t),
$$

where $p_{-1}(t)=0$. Then for each integer $i \in\{0,1, \ldots, D\}, p_{i}(t)=v_{0}(t)+v_{1}(t)+$ $\cdots+v_{i}(t)$ and the polynomial $p_{i}(t)$ has degree $i$, the leading coefficient $\left(c_{1} c_{2} \ldots c_{i}\right)^{-1}$. Moreover, $\left(t-\theta_{0}\right) p_{D}(t)=v_{D+1}(t)$ and for $i, j \in\{0,1, \ldots, D-1\}$

$$
\sum_{h=1}^{D} p_{i}\left(\theta_{h}\right) p_{j}\left(\theta_{h}\right)\left(\theta_{0}-\theta_{h}\right) m_{h}=\delta_{i, j} k_{i} b_{i}|X| .
$$

Lemma $3.2([23,48])$ Let $\theta$ be a real number such that $\theta \geq \theta_{1}$ or $\theta \leq \theta_{D}$. For every $\ell \in\{0,1, \ldots, D-1\}$, let

$$
g_{\ell}(t)=\sum_{h=0}^{\ell} \frac{b_{\ell} k_{\ell}}{b_{h} k_{h}} \frac{p_{h}(\theta)}{p_{\ell}(\theta)} p_{h}(t) .
$$

Then the following hold.

(i) If $\theta=\theta_{1}$ or $\theta_{D}$, then

$$
(t-\theta) g_{D-1}(t)=p_{D}(t)=\frac{1}{c_{1} c_{2} \cdots c_{D}}\left(t-\theta_{1}\right)\left(t-\theta_{2}\right) \cdots\left(t-\theta_{D}\right) .
$$

(ii) $p_{i}(t)=g_{i}(t)-\frac{b_{i}}{c_{i}} \frac{p_{i-1}(\theta)}{p_{i}(\theta)} g_{i-1}(t)$ for every $i \in\{0,1, \ldots, D-1\}$.

(iii) For every $\ell \in\{0,1, \ldots, D-2\}$

$$
\operatorname{tg}_{\ell}(t)=\beta_{\ell-1} g_{\ell-1}(t)+\alpha_{\ell} g_{\ell}(t)+\gamma_{\ell+1} g_{\ell+1}(t),
$$

where

$$
\begin{aligned}
\beta_{\ell-1} & =\frac{b_{\ell} c_{\ell+1}}{c_{\ell}} \frac{p_{\ell-1}(\theta) p_{\ell+1}(\theta)}{\left(p_{\ell}(\theta)\right)^{2}}, \\
\alpha_{\ell} & =a_{\ell}+c_{\ell}-c_{\ell+1}+\frac{p_{\ell-1}(\theta)}{p_{\ell}(\theta)} b_{\ell}-\frac{p_{\ell}(\theta)}{p_{\ell+1}(\theta)} b_{\ell+1}, \\
\gamma_{\ell+1} & =c_{\ell+1} .
\end{aligned}
$$

Moreover, if $\theta>\theta_{1}$ or $\theta<\theta_{D}$, then (19) holds for $\ell=D-1$ by setting $g_{D}(t)=p_{D}(t)$. 
(iv) For every $i, j \in\{0,1, \ldots, D-1\}$

$$
\sum_{h=1}^{D} g_{i}\left(\theta_{h}\right) g_{j}\left(\theta_{h}\right)\left(\theta-\theta_{h}\right)\left(\theta_{0}-\theta_{h}\right) m_{h}=\delta_{i, j} c_{i+1} \frac{p_{i+1}(\theta)}{p_{i}(\theta)} b_{i} k_{i}|X| .
$$

\section{Terwilliger algebras and their modules}

We now introduce the Terwilliger algebra of a distance-regular graph with respect to a subset of vertices.

Let $Y$ be a nonempty subset of the vertex set $X$ of a distance-regular graph $\Gamma$ of diameter $D$. The number

$$
w(Y)=\max \{\partial(x, y) \mid x, y \in Y\}
$$

is called the width of $Y$ in $\Gamma$. In particular, $w(X)=D$.

For $x \in X$, let

$$
\partial(x, Y)=\partial(Y, x)=\min \{\partial(x, y) \mid y \in Y\} .
$$

Set

$$
\tau=\tau(Y)=\max \{\partial(x, Y) \mid x \in X\} .
$$

The number $\tau$ is often called the covering radius of $Y$ in $X$. For $i \in\{0,1, \ldots, \tau\}$, let

$$
\Gamma_{i}(Y)=\{x \in X \mid \partial(x, Y)=i\},
$$

and let $E_{i}^{*}=E_{i}^{*}(Y)$ denote the diagonal matrix in $\operatorname{Mat}_{X}(\boldsymbol{C})$ with $(x, x)$-entry

$$
\left(E_{i}^{*}\right)_{x, x}= \begin{cases}1 & \text { if } x \in \Gamma_{i}(Y), \\ 0 & \text { otherwise }\end{cases}
$$

Throughout this paper, we adopt the convention that $E_{i}=0$ and $E_{j}^{*}=0$ for any integers $i$ and $j$ such that $i<0, j<0, i>D$ or $j>\tau$.

For a vector $\boldsymbol{v}=\sum_{x \in X} \alpha(x) \hat{x} \in V$ expressed as a linear combination of $\hat{x}$ 's, $\operatorname{supp}(\boldsymbol{v})$ denotes the support of $\boldsymbol{v}$, i.e., $\operatorname{supp}(\boldsymbol{v})=\{x \in X \mid \alpha(x) \neq 0\}$.

By definition, $E_{i}^{*} \boldsymbol{v}=\boldsymbol{v}$ if and only if $\operatorname{supp}(\boldsymbol{v}) \subset \Gamma_{i}(Y)$. Moreover, for a subset $Z$ of $X$, $\mathbf{1}_{Z}=\sum_{z \in Z} \hat{z}$ is called the characteristic vector of $Z$.

Lemma 4.1 For $h, i, j \in\{0,1, \ldots, D\}$ the following hold.

(i) $E_{i}^{*} E_{j}^{*}=\delta_{i, j} E_{i}^{*}$.

(ii) $E_{0}^{*}+E_{1}^{*}+\cdots+E_{D}^{*}=I$.

(iii) ${ }^{t}\left(E_{i}^{*}\right)=\overline{E_{i}^{*}}=E_{i}^{*}$. 
(iv) $E_{h}^{*} A_{i} E_{j}^{*} \neq 0$ if and only if there exist vertices $x \in \Gamma_{h}(Y)$ and $y \in \Gamma_{j}(Y)$ such that $\partial(x, y)=i$.

(v) If $E_{h}^{*} A_{i} E_{0}^{*} \neq 0$ then $h \leq i \leq h+w(Y)$.

Proof: Parts (i)-(iv) are immediate from the definition (20). For $x \in \Gamma_{h}(Y)$ and $y \in Y$, there exists $z \in Y$ such that $\partial(x, z)=h$ and $\partial(z, y) \leq w(Y)$. Hence $h \leq \partial(x, y) \leq h+w(Y)$. Thus (v) follows.

We have the decompositions

$$
\begin{aligned}
V & =E_{0} V+E_{1} V+\cdots+E_{D} V \text { (orthogonal direct sum), } \\
& =E_{0}^{*} V+E_{1}^{*} V+\cdots+E_{\tau}^{*} V \text { (orthogonal direct sum). }
\end{aligned}
$$

Definition 4.1 Let $\Gamma$ be a distance-regular graph. Let $X$ be the set of vertices and $Y$ a nonempty subset of $X$ such that $\tau=\tau(Y)$. Let $\mathcal{T}=\mathcal{T}(Y)$ denote the subalgebra of $\operatorname{Mat}_{X}(\boldsymbol{C})$ generated by the Bose-Mesner algebra $\mathcal{M}$ and $E_{0}^{*}, E_{1}^{*}, \ldots, E_{\tau}^{*}$. We call $\mathcal{T}$ the Terwilliger algebra (or subconstituent algebra) of $\Gamma$ with respect to $Y$.

Since $\mathcal{T}=\mathcal{T}(Y)$ is generated by symmetric real matrices, it is semisimple. The vector space $V$ is called the standard module of $\mathcal{T}$. It is a fact that every $\mathcal{T}$-module is isomorphic to a submodule of $V$. Thus, throughout this paper, the term $\mathcal{T}$-module shall refer only to vector subspaces of $V$ which are invariant under the action of $\mathcal{T}$ by the usual matrix multiplication.

Definition 4.2 Let $W$ be an irreducible $\mathcal{T}(Y)$-module. $W$ is said to be thin whenever

$$
\operatorname{dim} E_{i}^{*} W \leq 1 \quad \text { for all } i \in\{0,1, \ldots, D\}
$$

$W$ is said to be dual-thin whenever

$$
\operatorname{dim} E_{i} W \leq 1 \quad \text { for all } i \in\{0,1, \ldots, D\}
$$

Definition 4.3 Let $W$ be an irreducible $\mathcal{T}(Y)$-module. The endpoint $v$ and diameter $\delta$ of $W$ are the nonnegative integers defined by the following.

$$
v=\min \left\{i \mid E_{i}^{*} W \neq 0\right\}, v+\delta=\max \left\{i \mid E_{i}^{*} W \neq 0\right\} .
$$

Lemma 4.2 ([45, Lemma 3.9]) Let $\mathcal{T}=\mathcal{T}(Y)$ and let $W$ denote an irreducible $\mathcal{T}$-module of endpoint $v$ and diameter $\delta$. Then

$$
E_{j}^{*} W \neq 0 \quad \text { if and only if } \quad v \leq j \leq v+\delta .
$$

Moreover, the following hold. 
(i) $A E_{j}^{*} W \subset E_{j-1}^{*} W+E_{j}^{*} W+E_{j+1}^{*} W$ for every $j \in\{0,1, \ldots, D\}$.

(ii) $E_{i}^{*} A E_{j}^{*} W \neq 0$ if $|i-j|=1 \quad(v \leq i, j \leq v+\delta)$.

(iii) Suppose $W$ is thin. Then for every $i \in\{0,1, \ldots, \delta\}$

$$
E_{v}^{*} W+E_{v+1}^{*} W+\cdots+E_{v+i}^{*} W=E_{v}^{*} W+A E_{v}^{*} W+\cdots+A^{i} E_{v}^{*} W .
$$

(iv) Suppose $W$ is thin. Then for $j \in\{0,1, \ldots, D\}$

$$
E_{j} W=E_{j} E_{\nu}^{*} W
$$

Proof: Let $j \in\{0,1, \ldots, D\}$. By Lemma 4.1 (ii) and (iv), we have

$$
\begin{aligned}
A E_{j}^{*} W & =\sum_{i=0}^{D} E_{i}^{*} A E_{j}^{*} W \\
& =E_{j-1}^{*} A E_{j}^{*} W+E_{j}^{*} A E_{j}^{*} W+E_{j+1}^{*} A E_{j}^{*} W \subset E_{j-1}^{*} W+E_{j}^{*} W+E_{j+1}^{*} W
\end{aligned}
$$

Hence we have (i).

Let $v^{\prime}=\max \left\{i \mid E_{i}^{*} W \neq 0\right.$, and $\left.E_{i-1}^{*} A E_{i}^{*} W=0\right\}$. Let $\delta^{\prime}$ be the least nonnegative integer satisfying the following.

$$
E_{\nu^{\prime}+\delta^{\prime}}^{*} W \neq 0 \quad \text { and that } \quad E_{\nu^{\prime}+\delta^{\prime}+1}^{*} A E_{\nu^{\prime}+\delta^{\prime}}^{*} W=0
$$

Let $W^{\prime}=E_{v^{\prime}}^{*} W+E_{v^{\prime}+1}^{*} W+\cdots+E_{v^{\prime}+\delta^{\prime}}^{*} W$. Then $A W^{\prime} \subset W^{\prime}$ by (i) in this lemma. Since $E_{j}^{*} W^{\prime} \subset W^{\prime}$ for every $j \in\{0,1, \ldots, D\}, W^{\prime}$ is a non-zero $\mathcal{T}$-invariant subspace of $W$. Since $W$ is irreducible, we have $W=W^{\prime}$. This shows $v=v^{\prime}$ and $\delta=\delta^{\prime}$.

Certainly,

$$
E_{j+1}^{*} A E_{j}^{*} W \neq 0 \quad(v \leq j<v+\delta)
$$

and

$$
E_{j-1}^{*} A E_{j}^{*} W \neq 0 \quad(v<j \leq v+\delta)
$$

Thus we have (ii). In particular, we have $E_{j}^{*} W \neq 0$ if and only if $v \leq j \leq v+\delta$.

If $W$ is thin, the property (21) implies (iii). Thus we have $W=\mathcal{M} E_{v}^{*} W$. Multiplying both sides of this equation on the left by $E_{j}$ gives (iv).

Lemma 4.3 Let $\mathcal{T}=\mathcal{T}(Y)$ and let $W$ be an irreducible $\mathcal{T}$-module. Then the following are equivalent.

(i) For some vector $v, W=\mathcal{M v}$.

(ii) $W$ is dual thin. 
Moreover, if $W$ is thin, then $W$ is dual-thin and the vector $v$ above can be taken from $E_{v}^{*} W$, where $v$ is the endpoint of $W$.

Proof: Suppose $W=\mathcal{M} v$ for some vector $v \in V$. Then for every $i \in\{0,1, \ldots, D\}$,

$$
E_{i} W=E_{i} \mathcal{M} v \subset \operatorname{Span}\left(E_{i} v\right)
$$

Hence $W$ is dual thin.

Conversely, suppose that $W$ is dual thin. Then $\operatorname{dim} E_{i} W \leq 1$. Hence we can choose $\boldsymbol{v}_{i}$ so that $E_{i} W=\operatorname{Span}\left(\boldsymbol{v}_{i}\right)$ for every $i \in\{0,1, \ldots, D\}$. Let $\boldsymbol{v}=\boldsymbol{v}_{0}+\boldsymbol{v}_{1}+\cdots+\boldsymbol{v}_{D}$. Since $E_{i} \boldsymbol{v}=\boldsymbol{v}_{i}, E_{i} W \subset \mathcal{M} \boldsymbol{v}$ and $W \subset \mathcal{M} \boldsymbol{v}$. Therefore, $W=\mathcal{M} \boldsymbol{v}$.

If $W$ is thin, then $\operatorname{dim} E_{v}^{*} W=1$. Hence by Lemma 4.2 (iv) $W$ is dual thin and the last assertion is obvious.

\section{The significance of endpoint 0}

Let $Y$ be a nonempty subset of the vertex set $X$ of $\Gamma$, and let $\mathcal{T}=\mathcal{T}(Y)$. Let $W$ be a $\mathcal{T}$ module of endpoint $\nu$. In this section, we show that $W$ can be regarded as a $\mathcal{T}\left(Y_{v}\right)$-module of endpoint 0 , where $Y_{i}=\Gamma_{i}(Y)$. Hence, to study a single irreducible $\mathcal{T}$-module $W$, it is sufficient to study one of endpoint zero with respect to a suitable base subset. We continue to use the notation $E_{i}^{*}=E_{i}^{*}(Y)$ and $\tau=\tau(Y)$.

Lemma 5.1 The following hold.

(i) For each $x \in Y_{j}, \partial\left(x, Y_{i}\right)=j-i$ if $j \geq i$, and $i-j \leq \partial\left(x, Y_{i}\right)$ if $j<i$.

(ii) Fix $i \in\{0,1, \ldots, D\}$. Then for each $j \in\{0,1, \ldots, D\}$,

$$
E_{j}^{*}\left(Y_{i}\right)=E_{i+j}^{*}+E_{j}^{*}\left(Y_{i}\right)\left(E_{0}^{*}+E_{1}^{*}+\cdots+E_{i-1}^{*}\right) .
$$

Proof: (i) is obvious, and (ii) follows from (i).

Lemma 5.2 Let $V$ be the standard module of $\mathcal{T}$. For $v \in\{0,1, \ldots, \tau\}$, let $V(v)$ denote the sum of irreducible $\mathcal{T}$-modules of endpoint $v$. Then

$$
V=V(0)+V(1)+\cdots+V(\tau)(\text { orthogonal direct sum) } .
$$

Let $\mathcal{I}(v)$ be the annihilator of $U(v)=V(v)+V(v+1)+\cdots+V(\tau(Y))$ :

$$
\mathcal{I}(v)=\{M \in \mathcal{T} \mid M v=\mathbf{0} \text { for every } \boldsymbol{v} \in U(v)\}
$$

Then $\mathcal{T} / \mathcal{I}(v)$ is generated by the images of $A$ and $E_{v}^{*}, E_{v+1}^{*}, \ldots, E_{\tau}^{*}$. Moreover, if $W$ is an irreducible $\mathcal{T}$-module of endpoint $v$ then $W$ is a $\mathcal{T} / \mathcal{I}(v)$-module.

Proof: Since $\left\{E_{0}^{*}, E_{1}^{*}, \ldots, E_{v-1}^{*}\right\}$ is contained in the two sided ideal $\mathcal{I}(v)$ of $\mathcal{T}$, we have the assertion. 
Proposition 5.3 Let $U(v)$ be a $\mathcal{T}$-module defined in Lemma 5.2, and let $W$ be a $\mathcal{T}$ submodule of $U(v)$. Then the following hold.

(i) As linear transformations on $W, E_{i+v}^{*}$ and $E_{i}^{*}\left(Y_{v}\right)$ coincide for each $i \in\{0,1, \ldots, D-$ v).

(ii) $W$ is a $\mathcal{T}\left(Y_{v}\right)$-module of endpoint 0 .

(iii) $W$ is an irreducible $\mathcal{T}$-module if and only if it is an irreducible $\mathcal{T}\left(Y_{v}\right)$-module.

(iv) $W$ is a thin $\mathcal{T}$-module if and only if it is a thin $\mathcal{T}\left(Y_{v}\right)$-module.

Proof: Since $\left\{E_{0}^{*}, \ldots, E_{\nu-1}^{*}\right\}$ is contained in the annihilator of $U(v)$ and $W$ is contained in $U(v)$, (i) follows from Lemma 5.1. Now (ii), (iii), and (iv) follow immediately.

We end this section by a lemma related to another type of base change.

Lemma 5.4 Suppose $Z \subset Y \subset X$. Let $Z_{i}=\{x \in X \mid \partial(x, Z)=i\}$. Then the following hold.

(i) For every $x \in X, \partial(x, Z) \geq \partial(x, Y)$.

(ii) $Z_{i} \subset Y_{0} \cup Y_{1} \cup \cdots \cup Y_{i}$, and $Y_{i} \subset Z_{i} \cup Z_{i+1} \cup \cdots \cup Z_{D}$ for every $i \in\{0,1, \ldots, D\}$.

(iii) $E_{h}^{*}(Y) E_{\ell}^{*}(Z)=0$ for every $h, \ell \in\{0,1, \ldots, D\}$ such that $h>\ell$.

(iv) For every $i \in\{0,1, \ldots, D\}$,

$$
\begin{aligned}
& E_{i}^{*}(Z)=E_{0}^{*}(Y) E_{i}^{*}(Z)+\cdots+E_{i}^{*}(Y) E_{i}^{*}(Z), \quad \text { and } \\
& E_{i}^{*}(Y)=E_{i}^{*}(Z) E_{i}^{*}(Y)+\cdots+E_{D}^{*}(Z) E_{i}^{*}(Y) .
\end{aligned}
$$

Proof: (i) and (ii) are clear. (iii) and (iv) follow from (i) and (ii).

\section{Thin modules}

For the rest of the paper, we fix a nonempty subset $Y$ of the vertex set $X$ of $\Gamma$ unless otherwise noted. Let $\mathcal{T}=\mathcal{T}(Y)$ denote the Terwilliger algebra of $\Gamma$ with respect to $Y$, and $E_{i}^{*}=E_{i}^{*}(Y)$. Recall that $\mathcal{T}$ is generated by the Bose-Mesner algebra $\mathcal{M}$ of $\Gamma$ and $E_{i}^{*}$ $(i \in\{0,1, \ldots, D\})$ as an algebra.

Definition 6.1 Let $v \in V$ be a nonzero vector such that $\operatorname{supp}(v) \subset Y \subset X$, i.e., $\mathbf{0} \neq \boldsymbol{v} \in$ $E_{0}^{*} V$.

(i) Let $t=t(\boldsymbol{v})=\max \left\{i \mid E_{i}^{*} A_{i} \boldsymbol{v} \neq \mathbf{0}\right\}$.

(ii) Let $r=r(\boldsymbol{v})=\left|\left\{i \mid E_{i} \boldsymbol{v} \neq \mathbf{0}, i \in\{0,1, \ldots, D\}\right\}\right|-1$.

Remarks When $v$ is the characteristic vector of $Y, t(v)$ coincides with $\tau(Y)$. The number $r(v)$ is called the dual degree. See [2, Chapter 11]. 
Lemma 6.1 Let $v \in E_{0}^{*} V$ be a nonzero vector. Let $t=t(v)$ and $r=r(v)$. Then the following hold.

(i) $E_{i}^{*} A_{i} \boldsymbol{v} \neq \mathbf{0}$ for every $i \in\{0,1, \ldots, t\}$. In particular,

$$
t+1=\left|\left\{i \mid E_{i}^{*} A_{i} \boldsymbol{v} \neq \mathbf{0}\right\}\right| .
$$

(ii) $\operatorname{dim} \mathcal{M} v=r+1$ and

$$
\mathcal{M} \boldsymbol{v}=\operatorname{Span}\left(\boldsymbol{v}, A \boldsymbol{v}, \ldots, A^{r} \boldsymbol{v}\right)=\operatorname{Span}\left(A_{0} \boldsymbol{v}, A_{1} \boldsymbol{v}, \ldots, A_{r} \boldsymbol{v}\right) .
$$

(iii) $t(v) \leq r(v)$.

Proof: (i) By definition $E_{t}^{*} A_{t} \boldsymbol{v} \neq \mathbf{0}$. Let $i \in\{0,1, \ldots, t\}$. Then

$$
\mathbf{0} \neq p_{t-i, i}^{t} E_{t}^{*} A_{t} \boldsymbol{v}=E_{t}^{*} A_{t-i} A_{i} \boldsymbol{v}=E_{t}^{*} A_{t-i} E_{i}^{*} A_{i} \boldsymbol{v} .
$$

Hence $E_{i}^{*} A_{i} \boldsymbol{v} \neq \mathbf{0}$. By the choice of $t$, we have that $t+1=\left|\left\{i \mid E_{i}^{*} A_{i} \boldsymbol{v} \neq \mathbf{0}\right\}\right|$.

(ii) Observe that $\mathcal{M} \boldsymbol{v}=\operatorname{Span}\left(E_{0} \boldsymbol{v}, E_{1} \boldsymbol{v}, \ldots, E_{D} \boldsymbol{v}\right)$ and that nonzero vectors among $E_{0} v, E_{1} v, \ldots, E_{D} v$ are mutually orthogonal. Since nonzero mutually orthogonal vectors are linearly independent, $\operatorname{dim} \mathcal{M} v=r+1$ by the definition of $r(v)$. Since $\mathcal{M}$ is algebraically generated by the adjacency matrix $A, \mathcal{M} \boldsymbol{v}=C[A] \boldsymbol{v}$. Now $\mathcal{M} \boldsymbol{v}=\operatorname{Span}\left(\boldsymbol{v}, A \boldsymbol{v}, \ldots, A^{r} \boldsymbol{v}\right)$ follows as the polynomial ring over a field is a principal ideal domain. The last equality follows immediately.

(iii) By (ii) above and Lemma $4.1(\mathrm{v})$, for every $h>r$,

$$
E_{h}^{*} A_{h} v \in E_{h}^{*} \mathcal{M} \boldsymbol{v}=\operatorname{Span}\left(E_{h}^{*} A_{0} v, E_{h}^{*} A_{1} v, \ldots, E_{h}^{*} A_{r} v\right)=0
$$

Hence $t(v) \leq r(v)$.

Proposition 6.2 Let $v \in E_{0}^{*} V$ be a nonzero vector. Let $t=t(v)$. Then the following are equivalent.

(i) $\mathcal{T} v$ is a thin irreducible $\mathcal{T}$-module.

(ii) $\operatorname{dim} E_{i}^{*} \mathcal{M} v \leq 1$ for every $i \in\{0,1, \ldots, D\}$.

(iii) $E_{i}^{*} A_{i+1} v, E_{i}^{*} A_{i+2} v \in \operatorname{Span}\left(E_{i}^{*} A_{i} v\right)$ for every $i \in\{0,1, \ldots, t\}$.

(iv) $\operatorname{Span}\left(E_{0}^{*} \boldsymbol{v}, E_{1}^{*} A_{1} \boldsymbol{v}, \ldots, E_{t}^{*} A_{t} v\right)$ is A-invariant.

Suppose the equivalent conditions (i)-(iv) hold. Then

$$
\mathcal{T} \boldsymbol{v}=\mathcal{M} \boldsymbol{v}=\operatorname{Span}\left(E_{0}^{*} \boldsymbol{v}, E_{1}^{*} A_{1} v, \ldots, E_{t}^{*} A_{t} v\right)
$$

In particular, $r(\boldsymbol{v})=t(\boldsymbol{v})$ and $\mathcal{T} \boldsymbol{v}$ is of dimension $t+1$

Proof: Below we shall frequently use the fact that $E_{i}^{*} A_{j} \boldsymbol{v}=\mathbf{0}$ whenever $i>j$.

Let $\boldsymbol{v}_{i}=E_{i}^{*} A_{i} \boldsymbol{v}$ for all $i \in\{0,1, \ldots, D\}$. We assume that $\boldsymbol{v}_{j}=\mathbf{0}$ for all $j<0$ or $j>D$. 
(i) $\rightarrow$ (ii) Since $\mathcal{M} \subset \mathcal{T}$, if $\mathcal{T} v$ is thin irreducible, then for $i \in\{0,1, \ldots, D\}$ we have $\operatorname{dim} E_{i}^{*} \mathcal{M} \boldsymbol{v} \leq \operatorname{dim} E_{i}^{*} \mathcal{T} v \leq 1$

(ii) $\rightarrow$ (iii) This is obvious by the definition of $t(v)$ and Lemma 6.1 (i).

(iii) $\rightarrow$ (iv) Let $t=t(v)$ and $W_{0}=\operatorname{Span}\left(v_{0}, v_{1}, \ldots, v_{t}\right)$. By definition, $W_{0}$ is invariant under the actions of $E_{0}^{*}, E_{1}^{*}, \ldots, E_{D}^{*}$. Moreover, by our assumption,

$$
E_{i}^{*} A_{i+1} \boldsymbol{v}, E_{i}^{*} A_{i+2} \boldsymbol{v} \in \operatorname{Span}\left(\boldsymbol{v}_{i}\right) \quad \text { for every } i \in\{0,1, \ldots, t\} .
$$

We claim that $W_{0}$ is $A$-invariant. Let $0 \leq j \leq t$ and assume that for $h<j A v_{h} \in W_{0}$, or equivalently,

$$
E_{h+1}^{*} A v_{h}, \quad E_{h}^{*} A v_{h}, \quad E_{h-1}^{*} A v_{h} \in W_{0}
$$

Then we have by Lemma 4.2 (i), and (22), (23),

$$
\begin{aligned}
& A v_{j}=A E_{j}^{*} A_{j} v \\
& =E_{j+1}^{*} A E_{j}^{*} A_{j} v+E_{j}^{*} A E_{j}^{*} A_{j} v+E_{j-1}^{*} A E_{j}^{*} A_{j} v \\
& =E_{j+1}^{*} A A_{j} v+E_{j}^{*} A A_{j} v-E_{j}^{*} A E_{j-1}^{*} A_{j} v \\
& +E_{j-1}^{*} A A_{j} v-E_{j-1}^{*} A E_{j-1}^{*} A_{j} v-E_{j-1}^{*} A E_{j-2}^{*} A_{j} v \\
& =c_{j+1} E_{j+1}^{*} A_{j+1} v+c_{j+1} E_{j}^{*} A_{j+1} v+a_{j} E_{j}^{*} A_{j} v-E_{j}^{*} A E_{j-1}^{*} A_{j} v \\
& +c_{j+1} E_{j-1}^{*} A_{j+1} v+a_{j} E_{j-1}^{*} A_{j} v+b_{j-1} E_{j-1}^{*} A_{j-1} v \\
& -E_{j-1}^{*} A E_{j-1}^{*} A_{j} v-E_{j-1}^{*} A E_{j-2}^{*} A_{j} v \\
& \in \operatorname{Span}\left(\boldsymbol{v}_{j+1}, \boldsymbol{v}_{j}, \boldsymbol{v}_{j-1}, E_{j}^{*} A \boldsymbol{v}_{j-1}, E_{j-1}^{*} A \boldsymbol{v}_{j-1}, E_{j-1}^{*} A \boldsymbol{v}_{j-2}\right) \\
& \subset W_{0} \text {. }
\end{aligned}
$$

Now by induction, $W_{0}$ is invariant under the action of $A$, and hence $W_{0}$ is $\mathcal{T}$-invariant. Since $\boldsymbol{v} \in W_{0}, \mathcal{T} \boldsymbol{v}=W_{0}$ and we have (iv).

(iv) $\rightarrow$ (i) $\quad$ Let $W=\operatorname{Span}\left(\boldsymbol{v}_{0}, \boldsymbol{v}_{1}, \ldots, \boldsymbol{v}_{D}\right)$. Then $\boldsymbol{v}_{0}=\boldsymbol{v} \neq \mathbf{0}$ and $W \subset \mathcal{T} \boldsymbol{v}$. By definition, $W$ is invariant under the actions of $E_{0}^{*}, E_{1}^{*}, \ldots, E_{D}^{*}$. Moreover, by our assumption, $W$ is invariant under the action of $A$. Hence $W=\mathcal{T} v$. Let $W_{0}$ be an irreducible subconstituent of $W$ such that $E_{0}^{*} W_{0} \neq \mathbf{0}$. Since $E_{0}^{*} W=\operatorname{Span}\left(\boldsymbol{v}_{0}\right), \boldsymbol{v}=\boldsymbol{v}_{0} \in W_{0}$ and $W=\mathcal{T} \boldsymbol{v} \subset W_{0} \subset W$. Hence $W$ is irreducible. Moreover, since $E_{i}^{*} W \subset \operatorname{Span}\left(v_{i}\right), W$ is thin.

By (iv), $\mathcal{T} \boldsymbol{v}=\mathcal{M} \boldsymbol{v}=\operatorname{Span}\left(\boldsymbol{v}_{0}, \boldsymbol{v}_{1}, \ldots, \boldsymbol{v}_{t}\right)$, which is of dimension $t+1$ by the definition of $t(\boldsymbol{v})$. By Lemma 6.1 (ii), $\operatorname{dim} \mathcal{M} \boldsymbol{v}=r(\boldsymbol{v})+1$. Hence $r(\boldsymbol{v})=t(\boldsymbol{v})$. The last assertion follows from Lemma 4.3.

\section{The primary module}

In this section, we summarize the results concerning the module generated by the characteristic vector of $Y$. 
Let 1 be the all 1's vector of $V$. For every $i$, let

$$
\mathbf{1}_{i}=\sum_{x \in \Gamma_{i}(Y)} \hat{x}
$$

We have $\mathbf{1}=\mathbf{1}_{0}+\mathbf{1}_{1}+\cdots+\mathbf{1}_{\tau}$, where $\tau=\tau(Y)$. Note that $\mathbf{1}_{i}$ is the characteristic vector of $\Gamma_{i}(Y)$. In particular, $\mathbf{1}_{0}$ is the characteristic vector of the base subset $Y$.

Lemma 7.1 Let $W$ be an irreducible module of $\mathcal{T}$. Then the following are equivalent.

(i) $E_{0} W \neq 0$, i.e., $\mathbf{1} \in W$.

(ii) $\mathbf{1}_{0} \in W$.

(iii) $\mathbf{1}_{i} \in W$ for all $i \in\{0,1, \ldots, \tau\}$.

Proof: $\quad$ Since $E_{i}^{*} \mathbf{1}=\mathbf{1}_{i}$, (i) $\leftrightarrow$ (iii) and (i) $\rightarrow$ (ii) are immediate. Suppose (ii) holds. Then $(|Y| /|X|) \mathbf{1}=E_{0} \mathbf{1}_{0} \in W$ and (i) holds.

The irreducible module satisfying the conditions in the previous lemma is called the primary module of $\mathcal{T}$.

For $z \in \Gamma_{h}(Y)$, let

$$
\pi_{i, j}^{h}=\pi_{i, j}^{h}(z)=\left|\left\{y \in \Gamma_{i}(Y) \mid \partial(z, y)=j\right\}\right|=\left(E_{h}^{*} A_{j} \mathbf{1}_{i}\right)_{z} .
$$

Recall that a subset $Y$ of the vertex set $X$ of a distance-regular graph is called a completely regular code if $\pi_{0, j}^{h}(z)$ is independent of the choice of $z \in \Gamma_{h}(Y)$, i.e., $E_{h}^{*} A_{j} \mathbf{1}_{0}=\pi_{0, j}^{h} \mathbf{1}_{h}$ for all $h$ and $j$. Using Proposition 6.2, we can easily prove the following.

Proposition 7.2 Let $\mathbf{1}_{0}$ be the characteristic vector of the base subset $Y$ of $\mathcal{T}$. Then the following are equivalent.

(i) $Y$ is a completely regular code of $\Gamma$.

(ii) $\mathcal{T} \mathbf{1}_{0}$ is a thin irreducible $\mathcal{T}$-module.

(iii) $\mathcal{T} \mathbf{1}_{0}=\operatorname{Span}\left(\mathbf{1}_{0}, \mathbf{1}_{1}, \ldots, \mathbf{1}_{\tau}\right)$.

(iv) $E_{h}^{*} A_{j} \mathbf{1}_{i}=\pi_{i, j}^{h} \mathbf{1}_{h}$, i.e., $\pi_{i, j}^{h}(z)$ is independent of the choice of $z \in \Gamma_{h}(Y)$ for every $h, i, j \in\{0,1, \ldots, D\}$.

(v) Let $\beta_{j}=\pi_{j+1,1}^{j}, \alpha_{j}=\pi_{j, 1}^{j}$ and $\gamma_{j}=\pi_{j-1,1}^{j}$. Then for every $i \in\{0,1, \ldots, D\}$,

$$
A \mathbf{1}_{j}=\beta_{j-1} \mathbf{1}_{j-1}+\alpha_{j} \mathbf{1}_{j}+\gamma_{j+1} \mathbf{1}_{j+1} .
$$

Moreover, if one of these conditions are satisfied, then

$$
\begin{aligned}
\gamma_{j}\left|\Gamma_{j}(Y)\right| & =\beta_{j-1}\left|\Gamma_{j-1}(Y)\right| \text { and } \\
\left|\Gamma_{j}(Y)\right| & =\frac{\beta_{0} \beta_{1} \ldots \beta_{j-1}}{\gamma_{1} \gamma_{2} \ldots \gamma_{j}}|Y|, \quad \text { for all } j \in\{0,1,2, \ldots, \tau\} .
\end{aligned}
$$


Proof: Let $W=\mathcal{T} \mathbf{1}_{0}$ and $W_{0}=\operatorname{Span}\left(\mathbf{1}_{0}, \mathbf{1}_{1}, \ldots, \mathbf{1}_{\tau}\right)$. (i) is equivalent to $\mathcal{M} \mathbf{1}_{0} \subset W_{0}$. Hence Proposition 6.2 (i) (b) is satisfied. Thus (ii) holds. Other implications are also immediate.

\section{Remarks}

1. For definitions and algebraic characterizations of completely regular codes, see [2, Section 11.1] and [20, 21, 35].

2. If $Y=\{x\}$, then the primary module of $\mathcal{T}(Y)$ is always thin. It actually characterizes the property of a graph to be distance-regular with respect to a vertex $x$. See for example [42].

\section{Local eigenvalues}

Definition 8.1 For a nonzero vector $v \in V=C^{X}$, let

$$
\eta^{(i)}(\boldsymbol{v})=\frac{{ }^{t} \boldsymbol{v} A_{i} \overline{\boldsymbol{v}}}{{ }^{t} \bar{v} \overline{\boldsymbol{v}}}, \quad \text { for } i \in\{0,1, \ldots, D\}
$$

Let $\tilde{A}_{i}=E_{0}^{*} A_{i} E_{0}^{*}$ for $i \in\{0,1, \ldots, D\}$. Suppose $\operatorname{supp}(v) \subset Y$, i.e., $v \in E_{0}^{*} V$. Since $\boldsymbol{v}=E_{0}^{*} \boldsymbol{v}$

$$
{ }^{t} \boldsymbol{v} A_{i} \overline{\boldsymbol{v}}={ }^{t} \boldsymbol{v} E_{0}^{*} A_{i} E_{0}^{*} \overline{\boldsymbol{v}}={ }^{t} \boldsymbol{v} \tilde{A}_{i} \overline{\boldsymbol{v}}
$$

and $\eta^{(i)}(\boldsymbol{v})=0$ for every $i>w(Y)$ as $\tilde{A}_{i}=0$. Moreover if $\boldsymbol{v}$ is an eigenvector of $\tilde{A}_{i}$ for some $i \in\{0,1, \ldots, D\}$, then $\eta^{(i)}(v)$ is the eigenvalue of $\tilde{A}_{i}$ associated with the eigenvector $\boldsymbol{v}$ as $\boldsymbol{v}=E_{0}^{*} \boldsymbol{v}$.

Lemma 8.1 Let $W$ be a thin irreducible $\mathcal{T}$-module of endpoint 0 . Let $\boldsymbol{v}$ be a nonzero vector in $E_{0}^{*} W$, and $\eta^{(i)}=\eta^{(i)}(\boldsymbol{v})$ for each $i$. Then $E_{0}^{*} W=\operatorname{Span}(\boldsymbol{v})$ and $\boldsymbol{v}$ is a common eigenvector of $\tilde{A}_{0}, \tilde{A}_{1}, \ldots, \tilde{A}_{D}$. In particular, $\tilde{A}_{i} v=\eta^{(i)} v$ for every $i \in\{0,1, \ldots, D\}$.

Proof: Since $W$ is thin, $E_{0}^{*} W \neq 0$ is of dimension 1 . Hence it is spanned by $v$ and we have the following.

$$
\tilde{A}_{i} \boldsymbol{v}=E_{0}^{*} A_{i} E_{0}^{*} \boldsymbol{v} \in E_{0}^{*} W=\operatorname{Span}(\boldsymbol{v}) .
$$

Hence we have the desired conclusion.

Lemma 8.2 Let $v \in E_{0}^{*} V$ be a nonzero vector, and let $v_{j}(t)(j=0,1, \ldots, D)$ be the polynomials in Lemma 3.1. Then

$$
\frac{\left\|E_{i} \boldsymbol{v}\right\|^{2}}{\|\boldsymbol{v}\|^{2}}=\frac{1}{|X|} \sum_{j=0}^{w(Y)} \eta^{(j)}(\boldsymbol{v}) q_{i}(j)=\frac{1}{|X|} \sum_{j=0}^{w(Y)} \frac{\eta^{(j)}(\boldsymbol{v})}{k_{j}} v_{j}\left(\theta_{i}\right) m_{i}
$$


Proof: Set $\eta^{(i)}=\eta^{(i)}(\boldsymbol{v})$. Since $v_{j}\left(\theta_{i}\right)$ is the eigenvalue of $A_{j}$ on $E_{i} V, v_{j}\left(\theta_{i}\right)=p_{j}(i)$. See Lemma 3.1 (i), and (9). Hence by (11),

$$
q_{i}(j)=\frac{m_{i} v_{j}\left(\theta_{i}\right)}{k_{j}}
$$

Now using (10) and the remark following Definition 8.1, we have

$$
\begin{aligned}
\left\|E_{i} \boldsymbol{v}\right\|^{2} & ={ }^{t} \boldsymbol{v}^{t} E_{i} \overline{E_{i}} \overline{\boldsymbol{v}} \\
& ={ }^{t} \boldsymbol{v} E_{i} \overline{\boldsymbol{v}} \\
& ={ }^{t} \boldsymbol{v} \frac{1}{|X|} \sum_{j=0}^{D} q_{i}(j) A_{j} \overline{\boldsymbol{v}} \\
& =\frac{1}{|X|} \sum_{j=0}^{D} q_{i}(j)^{t} \boldsymbol{v} A_{j} \overline{\boldsymbol{v}} \\
& =\frac{1}{|X|} \sum_{j=0}^{w(Y)} \eta^{(j)} q_{i}(j)\|\boldsymbol{v}\|^{2} \\
& =\frac{1}{|X|} \sum_{j=0}^{w(Y)} \frac{\eta^{(j)}}{k_{j}} v_{j}\left(\theta_{i}\right) m_{i}\|\boldsymbol{v}\|^{2} .
\end{aligned}
$$

\section{The subspace $\mathcal{M} v$}

Definition 9.1 Let $v \in V$ be a nonzero vector. Let

$$
\rho_{\boldsymbol{v}}(t)=\frac{1}{|X|} \sum_{j=0}^{D} \eta^{(j)}(\boldsymbol{v}) \frac{v_{j}(t)}{k_{j}}
$$

where $v_{j}(t)$ is a polynomial in $\boldsymbol{R}[t]$ of degree $j$ such that $v_{j}(A)=A_{j}$. See Lemma 3.1.

Note that if in addition $v \in E_{0}^{*} V$, i.e., $\operatorname{supp}(v) \subset Y$, then

$$
\rho_{\boldsymbol{v}}(t)=\frac{1}{|X|} \sum_{j=0}^{w(Y)} \eta^{(j)}(\boldsymbol{v}) \frac{v_{j}(t)}{k_{j}}
$$

and $\operatorname{deg} \rho_{\boldsymbol{v}}(t) \leq w(Y)$.

Let $\Theta=\left\{\theta_{0}, \theta_{1}, \theta_{2}, \ldots, \theta_{D}\right\}$ and let $m$ be a function on $\Theta$ such that $m\left(\theta_{i}\right)=m_{i}$. Then $\left\{v_{0}(t), v_{1}(t), \ldots, v_{D}(t)\right\}$ is a system of orthogonal polynomials associated with the weight function $m$ on $\Theta$ satisfying the following. See Lemma 3.1.

$$
\left\langle v_{i}(t), v_{j}(t)\right\rangle_{m}=\sum_{\theta \in \Theta} v_{i}(\theta) v_{j}(\theta) m(\theta)=\delta_{i, j}|X| k_{i}
$$


for every $i, j \in\{0,1,2, \ldots, D\}$. Moreover, it follows from Lemma 8.2 that

$$
\frac{\left\|E_{i} \boldsymbol{v}\right\|^{2}}{\|\boldsymbol{v}\|^{2}}=\rho_{\boldsymbol{v}}\left(\theta_{i}\right) m\left(\theta_{i}\right) \geq 0 \quad \text { for every } i \in\{0,1,2, \ldots, D\}
$$

Proposition 9.1 Let $v$ be a nonzero vector in $E_{0}^{*} V$. Then

$$
r(\boldsymbol{v})=D-\left|\left\{\theta \in \Theta \mid \rho_{\boldsymbol{v}}(\theta)=0\right\}\right| \geq D-\operatorname{deg} \rho_{\boldsymbol{v}}(t) \geq D-w(Y) .
$$

Proof: This is immediate from Definition 6.1 and (25).

Definition 9.2 A nonzero vector $v \in E_{0}^{*} V$ is said to be tight with respect to $Y$, if $r(v)=$ $D-w(Y)$.

Theorem 9.2 Let $v$ be a nonzero vector in $E_{0}^{*} V$. If $v$ is tight with respect to $Y$, then $\mathcal{M} v$ is a thin irreducible $\mathcal{T}$-module. In particular, $\boldsymbol{v}$ is a common eigenvector of $\widetilde{\mathcal{M}}=E_{0}^{*} \mathcal{M} E_{0}^{*}$.

Proof: Let $w=w(Y)$ and $\rho(t)=\rho_{\boldsymbol{v}}(t)$. By (25) above, $\rho(\theta) \geq 0$ for every eigenvalue of the adjacency matrix $A$. Suppose $\theta_{i_{1}}, \theta_{i_{2}}, \ldots, \theta_{i_{w}}$ are the roots of $\rho(t)$. Hence there is a nonzero constant $c$ such that

$$
\rho(t)=c\left(t-\theta_{i_{1}}\right)\left(t-\theta_{i_{2}}\right) \cdots\left(t-\theta_{i_{w}}\right) .
$$

Then we have

$$
E_{i_{1}} v=E_{i_{2}} v=\cdots=E_{i_{w}} v=\mathbf{0}
$$

Hence for every $n \in\{0,1, \ldots, D-w\}$,

$$
E_{n}^{*} E_{i_{1}} \boldsymbol{v}=E_{n}^{*} E_{i_{2}} \boldsymbol{v}=\cdots=E_{n}^{*} E_{i_{w}} \boldsymbol{v}=\mathbf{0} .
$$

Thus for every $j \in\{1,2, \ldots, w\}$ we have

$$
\begin{aligned}
\mathbf{0} & =E_{n}^{*} E_{i_{j}} \boldsymbol{v} \\
& =\frac{m\left(\theta_{i_{j}}\right)}{|X|} \sum_{h=0}^{D} \frac{v_{h}\left(\theta_{i_{j}}\right)}{k_{h}} E_{n}^{*} A_{h} \boldsymbol{v} \\
& =\frac{m\left(\theta_{i_{j}}\right)}{|X|} \sum_{h=0}^{w} \frac{v_{n+h}\left(\theta_{i_{j}}\right)}{k_{n+h}} E_{n}^{*} A_{n+h} \boldsymbol{v},
\end{aligned}
$$

where we used Lemma 4.1 (v). 
Hence for each $j \in\{0,1, \ldots, w\}$, we have a linear equation

$$
\sum_{h=1}^{w} v_{n+h}\left(\theta_{i_{j}}\right) \frac{1}{k_{n+h}} E_{n}^{*} A_{n+h} \boldsymbol{v}=-\frac{v_{n}\left(\theta_{i_{j}}\right)}{k_{n}} E_{n}^{*} A_{n} \boldsymbol{v} .
$$

By Lemma 2.3,

$$
D_{\rho, n+1}=\left|\begin{array}{cccc}
v_{n+1}\left(\theta_{i_{1}}\right) & v_{n+2}\left(\theta_{i_{1}}\right) & \cdots & v_{n+w}\left(\theta_{i_{1}}\right) \\
v_{n+1}\left(\theta_{i_{2}}\right) & v_{n+2}\left(\theta_{i_{2}}\right) & \cdots & v_{n+w}\left(\theta_{i_{2}}\right) \\
\ldots \ldots \ldots \ldots \ldots \ldots \ldots \ldots \ldots \ldots \ldots \ldots \ldots \ldots \ldots \ldots \ldots & \ldots \ldots \ldots
\end{array}\right| \neq 0 .
$$

Hence for every $h \in\{0,1, \ldots, w\}, \frac{1}{k_{n+h}} E_{n}^{*} A_{n+h} \boldsymbol{v}$, and therefore $E_{n}^{*} A_{n+h} \boldsymbol{v}$ is a scalar multiple of $E_{n}^{*} A_{n} \boldsymbol{v}$. Since $E_{n}^{*} A_{n+h} \boldsymbol{v} \neq \mathbf{0}$ only when $h \in\{0,1, \ldots, w\}$, we have shown the conditions in Proposition 6.2 (iii). Hence by the same proposition, $\mathcal{T} \boldsymbol{v}$ is a thin irreducible $\mathcal{T}$-module as desired. The last assertion follows directly from our proof above. See also Lemma 8.1.

Corollary 9.3 Let $Y$ be a subset of the vertex set $X$ of a distance-regular graph. Suppose $w=w(Y)$ and for $i \in\{0,1, \ldots, D\}$ set

$$
\kappa_{i}=\frac{{ }^{t} \mathbf{1}_{0} A_{i} \mathbf{1}_{0}}{{ }^{t} \mathbf{1}_{0} \mathbf{1}_{0}}=\frac{1}{|Y|}\left|\left\{\left(y_{1}, y_{2}\right) \in Y \times Y \mid \partial\left(y_{1}, y_{2}\right)=i\right\}\right|,
$$

where $\mathbf{1}_{0}$ is the characteristic vector of $Y$. Let $\rho(t)=\rho \mathbf{1}_{0}(t)$. Then the following hold.

(i) $\rho(\theta) \geq 0$ for every eigenvalue $\theta$ of the adjacency matrix of $\Gamma$, where $\rho(t)$ is the following polynomial.

$$
\rho(t)=\frac{1}{|X|} \sum_{i=0}^{w} \frac{\kappa_{i}}{k_{i}} v_{i}(t)
$$

(ii) If $\rho(t)$ has $w$ roots in the set of eigenvalues of the adjacency matrix of $\Gamma$, then $Y$ is completely regular.

Proof: The assertions follow directly from Theorem 9.2.

Remarks Corollary 9.3 can be viewed as a generalization of the Hoffman bound of the size of a clique and the condition for equality, which can be derived easily by setting $w(Y)=1$. See Lemma 11.1. Similar conditions were studied in [20, 21]. It would be interesting to further consider the case where $E_{0}^{*} V$ is spanned by tight vectors. 


\section{Thin conditions}

In this section, we consider thin conditions. Let $r=r(v)$ and

$$
\Theta(v)=\left\{\theta \in \Theta \mid \rho_{\boldsymbol{v}}(\theta) \neq 0\right\}
$$

of cardinality $r+1$. Then by Proposition 2.1, there is a system of orthogonal polynomials $\left\{g_{0}(t), g_{1}(t), \ldots, g_{r}(t)\right\}$ associated with the weight function $\omega=\rho \cdot m$ on $\Theta(v)$ with $\rho=\rho \boldsymbol{v}$. We may assume that the leading coefficient of $g_{i}(t)$ coincides with that of $v_{i}(t)$. Then this system of polynomials is unique and there exist $c_{i}^{(j)} \in \boldsymbol{R}$ for $j \in\{0,1, \ldots, i\}$ such that

$$
g_{i}(t)=\sum_{j=0}^{i} c_{i}^{(j)} v_{j}(t),
$$

with $c_{i}^{(i)}=1$. Set

$$
g_{r+1}(t)=\prod_{\theta \in \Theta(\boldsymbol{v})}(t-\theta)
$$

Then for each $i \in\{0,1, \ldots, r\}$ there exist real numbers $\beta_{i-1}, \alpha_{i}, \gamma_{i+1}$ and $g_{0}(t), g_{1}(t), \ldots$, $g_{r}(t), g_{r+1}(t)$ satisfy the following relations.

$$
t_{i}(t)=\beta_{i-1} g_{i-1}(t)+\alpha_{i} g_{i}(t)+\gamma_{i+1} g_{i+1}(t) .
$$

Moreover, $\beta_{i-1} \gamma_{i}>0$ for $i \in\{1,2, \ldots, r\}$.

Theorem 10.1 Let $\boldsymbol{v}$ be a nonzero vector in $E_{0}^{*} V$, and let $\boldsymbol{u}_{i}=g_{i}(A) v$ for $i \in\{0,1, \ldots, r+$ 1\}. Then the following hold.

(i) For every $i, j \in\{0,1, \ldots, r+1\}$,

$$
\left\langle\boldsymbol{u}_{i}, \boldsymbol{u}_{j}\right\rangle=\left\langle g_{i}(t), g_{j}(t)\right\rangle_{\omega}\langle\boldsymbol{v}, \boldsymbol{v}\rangle=\delta_{i, j}\left\langle g_{i}(t), g_{i}(t)\right\rangle_{\omega}\|\boldsymbol{v}\|^{2},
$$

and $\boldsymbol{u}_{r+1}=\mathbf{0}$.

(ii) For every $i \in\{0,1, \ldots, r\}$,

$$
\left\|E_{i}^{*} A_{i} \boldsymbol{v}\right\|^{2} \leq\left\|\boldsymbol{u}_{i}\right\|^{2} .
$$

Moreover, equality holds above if and only if $\boldsymbol{u}_{i} \in E_{i}^{*} V$. In this case, $\boldsymbol{u}_{i}=E_{i}^{*} A_{i} \boldsymbol{v}$.

(iii) The following are equivalent.

(a) $\mathcal{T}$-module $\mathcal{T} \boldsymbol{v}$ is thin and irreducible.

(b) Equality holds in (ii) for every $i \in\{0,1, \ldots, r\}$.

(c) Equality holds in (ii) for $i=r$. 
Proof: (i) By (25), $\left\|E_{h} \boldsymbol{v}\right\|^{2}=\rho\left(\theta_{h}\right) m\left(\theta_{h}\right)\|\boldsymbol{v}\|^{2}$. Hence

$$
\begin{aligned}
\left\langle\boldsymbol{u}_{i}, \boldsymbol{u}_{j}\right\rangle & =\left\langle g_{i}(A) \boldsymbol{v}, g_{j}(A) \boldsymbol{v}\right\rangle \\
& =\left\langle g_{i}(A) \sum_{h=0}^{D} E_{h} \boldsymbol{v}, g_{j}(A) \sum_{h=0}^{D} E_{h} \boldsymbol{v}\right\rangle \\
& =\left\langle g_{i}(A) \sum_{\theta_{h} \in \Theta(\boldsymbol{v})} E_{h} \boldsymbol{v}, g_{j}(A) \sum_{\theta_{h} \in \Theta(\boldsymbol{v})} E_{h} \boldsymbol{v}\right\rangle \\
& =\left\langle\sum_{\theta_{h} \in \Theta(\boldsymbol{v})} g_{i}\left(\theta_{h}\right) E_{h} \boldsymbol{v}, \sum_{\theta_{h} \in \Theta(\boldsymbol{v})} g_{j}\left(\theta_{h}\right) E_{h} \boldsymbol{v}\right\rangle \\
& =\sum_{\theta_{h} \in \Theta(\boldsymbol{v})} g_{i}\left(\theta_{h}\right) g_{j}\left(\theta_{h}\right) \rho\left(\theta_{h}\right) m\left(\theta_{h}\right)\langle\boldsymbol{v}, \boldsymbol{v}\rangle \\
& =\sum_{\theta_{h} \in \Theta(\boldsymbol{v})} g_{i}\left(\theta_{h}\right) g_{j}\left(\theta_{h}\right) \omega\left(\theta_{h}\right)\langle\boldsymbol{v}, \boldsymbol{v}\rangle \\
& =\left\langle g_{i}(t), g_{j}(t)\right\rangle_{\omega}\langle\boldsymbol{v}, \boldsymbol{v}\rangle \\
& =\delta_{i, j}\left\langle g_{i}(t), g_{i}(t)\right\rangle_{\omega}\|\boldsymbol{v}\|^{2} .
\end{aligned}
$$

Since $g_{r+1}(t)$ is a zero function on $\Theta(\boldsymbol{v}), \boldsymbol{u}_{r+1}=\mathbf{0}$.

(ii) By the definition of $r=r(\boldsymbol{v}), \boldsymbol{u}_{i} \neq \mathbf{0}$ for $i \in\{0,1, \ldots, r\}$. Note that $g_{i}(t)$ is of degree $i$ and the leading coefficient of $g_{i}(t)$ coincides with that of $v_{i}(t)$ by definition. Hence if $g_{i}(A)$ is expressed as a linear combination of $A_{0}, A_{1}, \ldots, A_{D}$, then $A_{j}$ with $j>i$ does not appear and the coefficient of $A_{i}$ is 1 . Hence $E_{i}^{*} g_{i}(A) \boldsymbol{v}=E_{i}^{*} A_{i} \boldsymbol{v}$ and

$$
\boldsymbol{u}_{i}=g_{i}(A) \boldsymbol{v}=E_{i}^{*} A_{i} \boldsymbol{v}+\sum_{h=0}^{i-1} E_{h}^{*} g_{i}(A) \boldsymbol{v} .
$$

Since the vector appearing in the right hand side of the equation are mutually orthogonal, $\left\|E_{i}^{*} A_{i} \boldsymbol{v}\right\|^{2} \leq\left\|\boldsymbol{u}_{i}\right\|^{2}$. Hence the first assertion follows from (i). Moreover, $\boldsymbol{u}_{i} \in E_{i}^{*} V$ if and only if $\sum_{h=0}^{i-1} E_{h}^{*} g_{i}(A) \boldsymbol{v}=\mathbf{0}$. Thus we have (ii).

(iii) (a) $\rightarrow$ (b) Suppose $W=\mathcal{T} v$ is thin and irreducible. By Proposition $6.2 r(v)=t(v)$. Hence $W=\mathcal{M} \boldsymbol{v}$ and $W$ is an orthogonal direct sum of $E_{i}^{*} W=\operatorname{Span}\left(E_{i}^{*} A_{i} v\right)$. Set $\boldsymbol{v}_{i}=$ $E_{i}^{*} A_{i} \boldsymbol{v}$ for $i=0,1, \ldots, r$. Then $\left\{\boldsymbol{v}_{0}, \boldsymbol{v}_{1}, \ldots, \boldsymbol{v}_{r}\right\}$ forms a mutually orthogonal basis. On the other hand, since $\boldsymbol{u}_{0}, \boldsymbol{u}_{1}, \ldots, \boldsymbol{u}_{r}$ are mutually orthogonal and gives a basis of $\mathcal{M} \boldsymbol{v}$, we have the following.

$$
\boldsymbol{v}_{i} \in E_{i}^{*} W \subset E_{i}^{*}\left(\operatorname{Span}\left(\boldsymbol{u}_{i}, \boldsymbol{u}_{i+1}, \ldots, \boldsymbol{u}_{r}\right) \cap \operatorname{Span}\left(\boldsymbol{v}_{i+1}, \ldots, \boldsymbol{v}_{r}\right)^{\perp}\right) .
$$

We prove by induction on $i$ in reverse order that $\operatorname{Span}\left(\boldsymbol{u}_{i}\right)=\operatorname{Span}\left(\boldsymbol{v}_{i}\right)$. Suppose the assertion holds for $j>i$ for $i \in\{0,1, \ldots, r\}$. By the inductive hypothesis,

$$
\operatorname{Span}\left(\boldsymbol{u}_{i+1}, \ldots, \boldsymbol{u}_{r}\right)=\operatorname{Span}\left(\boldsymbol{v}_{i+1}, \ldots, \boldsymbol{v}_{r}\right) .
$$


Hence by (28) and (29),

$$
\begin{aligned}
\mathbf{0} \neq \boldsymbol{v}_{i} & \in \operatorname{Span}\left(E_{i}^{*} \boldsymbol{u}_{i}\right) \\
& \subset \operatorname{Span}\left(\boldsymbol{u}_{0}, \boldsymbol{u}_{1}, \ldots, \boldsymbol{u}_{i-1}\right)^{\perp} \cap \operatorname{Span}\left(\boldsymbol{v}_{i+1}, \ldots, \boldsymbol{v}_{r}\right)^{\perp} \cap W \\
& \subset \operatorname{Span}\left(\boldsymbol{u}_{i}, \boldsymbol{u}_{i+1}, \ldots, \boldsymbol{u}_{r}\right) \cap \operatorname{Span}\left(\boldsymbol{u}_{i+1}, \ldots, \boldsymbol{u}_{r}\right)^{\perp} \\
& \subset \operatorname{Span}\left(\boldsymbol{u}_{i}\right)
\end{aligned}
$$

By (ii), we have the assertion.

(b) $\rightarrow$ (c) This is obvious.

(c) $\rightarrow$ (b) By (ii), we assume that $\boldsymbol{u}_{r} \in \operatorname{Span}\left(E_{r}^{*} A_{r} \boldsymbol{v}\right)$. Since $\boldsymbol{u}_{r+1}=\mathbf{0}$, by (27) we have

$$
A \boldsymbol{u}_{i}=\beta_{i-1} \boldsymbol{u}_{i-1}+\alpha_{i} \boldsymbol{u}_{i}+\gamma_{i+1} \boldsymbol{u}_{i+1}
$$

for every $i \in\{0,1, \ldots, r\}$. Since $\operatorname{deg} g_{i}(t)=i$,

$$
\boldsymbol{u}_{i} \in E_{0}^{*} V+E_{1}^{*} V+\cdots+E_{i}^{*} V
$$

for every $i \in\{0,1, \ldots, r\}$. Suppose $\boldsymbol{u}_{i} \in E_{i}^{*} V$ for $i \leq r$. Then by (30)

$$
\beta_{i-1} \boldsymbol{u}_{i-1} \in\left(E_{i-1}^{*} V+\cdots+E_{r}^{*} V\right) \cap\left(E_{0}^{*} V+E_{1}^{*} V+\cdots+E_{i-1}^{*} V\right)=E_{i-1}^{*} V .
$$

Since $\beta_{i-1} \neq 0$ for $i \in\{1,2, \ldots, r\}$, we have $\boldsymbol{u}_{i-1} \in E_{i-1}^{*} V$. Thus we have (b) by induction.

(b) $\rightarrow$ (a) Suppose $\boldsymbol{u}_{i} \in \operatorname{Span}\left(\boldsymbol{v}_{i}\right)$ for every $i \in\{0,1, \ldots, r\}$, then $r(v)=t(v)$ and

$$
W=\operatorname{Span}\left(\boldsymbol{u}_{0}, \boldsymbol{u}_{1}, \ldots, \boldsymbol{u}_{r}\right)=\operatorname{Span}\left(\boldsymbol{v}_{0}, \boldsymbol{v}_{1}, \ldots, \boldsymbol{v}_{t}\right)
$$

Hence $W$ is $A$-invariant and it is also stable under the actions of $E_{0}^{*}, E_{1}^{*}, \ldots, E_{D}^{*}$. Thus $W=\mathcal{T} \boldsymbol{v}$ and it is irreducible and thin by Proposition 6.2.

Corollary 10.2 Let $v$ be a nonzero vector in $E_{0}^{*} V$. Let $r=r(v)$. Then the condition whether $\mathcal{T} \boldsymbol{v}$ is a thin irreducible $\mathcal{T}$-module is determined by $\eta^{(i)}(\boldsymbol{v}), i \in\{0,1, \ldots, D\}$, and $\left\|E_{r}^{*} A_{r} v\right\| / \mid v \|$ together with the parameters of $\Gamma$.

Proof: By Theorem 10.1, $\mathcal{T} v$ is thin and irreducible if and only if

$$
\left\|E_{r}^{*} A_{r} v\right\|^{2} /\|v\|^{2}=<g_{r}(t), g_{r}(t)>_{\rho m} .
$$

Now the assertion follows immediately.

Corollary 10.3 Let $W_{1}$ and $W_{2}$ are irreducible thin $\mathcal{T}$-modules. Then $W_{1}$ and $W_{2}$ are isomorphic if and only if the endpoints $v$ are same and $\rho_{\boldsymbol{w}_{1}}(t)=\rho_{\boldsymbol{w}_{2}}(t)$, where $E_{v}^{*} W_{1}=$ $\operatorname{Span}\left(\boldsymbol{w}_{1}\right)$ and $E_{v}^{*} W_{2}=\operatorname{Span}\left(\boldsymbol{w}_{2}\right)$. 
Proof: By Proposition 5.3 we may assume that $v=0$. Suppose $\rho_{\boldsymbol{w}_{1}}(t)=\rho_{\boldsymbol{w}_{2}}(t)$. Then the module structure is determined by the system of orthogonal polynomials defined above. Hence they are isomorphic.

Conversely, if $W_{1}$ and $W_{2}$ are isomorphic, $\eta^{(i)}\left(\boldsymbol{w}_{1}\right)=\eta^{(i)}\left(\boldsymbol{w}_{2}\right)$ for every $i \in\{0,1, \ldots, D\}$ by Lemma 8.1 . Hence the polynomials $\rho_{\boldsymbol{w}_{1}}(t), \rho_{\boldsymbol{w}_{2}}(t)$ defined by each vector are equal.

We are now ready to prove Theorems 1.1 and 1.2.

Proof of Theorems 1.1 and 1.2: Theorem 1.1 (i) is a direct consequence of Proposition 9.1 together with the fact that $\operatorname{dim} \mathcal{M} \boldsymbol{v}=r(\boldsymbol{v})+1$ proved in Lemma 6.1 (ii). Suppose $r(\boldsymbol{v})=$ $D-w(Y)$. Then $v$ is tight and by Theorem $9.2 \mathcal{T} v$ is a thin irreducible module.

Theorem 1.2 follows from Theorem 10.1 (iii).

The following is also obtained as an application of Theorem 10.1.

Proposition 10.4 Let $v \in V=C^{X}$ be a nonzero vector. Let $\operatorname{supp}(v) \subset Z \subset Y \subset X$. Then the following hold.

(i) $E_{i}^{*}(Y) A_{i} \boldsymbol{v}=E_{i}^{*}(Y) E_{i}^{*}(Z) A_{i} \boldsymbol{v}$, and $\left\|E_{i}^{*}(Y) A_{i} \boldsymbol{v}\right\| \leq\left\|E_{i}^{*}(Z) A_{i} \boldsymbol{v}\right\|$ for every $i \in\{0,1$, $\ldots, D\}$. Equality holds if and only if $E_{i}^{*}(Y) A_{i} v=E_{i}^{*}(Z) A_{i} v$.

(ii) If $W=\mathcal{T}(Y) v$ is a thin irreducible $\mathcal{T}(Y)$-module, then $W$ is a thin irreducible $\mathcal{T}(Z)$ module.

Proof: (i) By Lemma 5.4 (iii), $E_{i}^{*}(Y) E_{j}^{*}(Z)=0$ if $i>j$. Hence

$$
\begin{aligned}
E_{i}^{*}(Y) A_{i} \boldsymbol{v} & =\sum_{j=0}^{i-1} E_{i}^{*}(Y) E_{j}^{*}(Z) A_{i} \boldsymbol{v}+E_{i}^{*}(Y) E_{i}^{*}(Z) A_{i} \boldsymbol{v}+\sum_{j=i+1}^{D} E_{i}^{*}(Y) E_{j}^{*}(Z) A_{i} \boldsymbol{v} \\
& =E_{i}^{*}(Y) E_{i}^{*}(Z) A_{i} \boldsymbol{v} .
\end{aligned}
$$

Therefore,

$$
\begin{aligned}
E_{i}^{*}(Z) A_{i} \boldsymbol{v} & =\left(E_{0}^{*}(Y) E_{i}^{*}(Z)+\cdots+E_{i}^{*}(Y) E_{i}^{*}(Z)\right) A_{i} \boldsymbol{v} \\
& =\left(E_{0}^{*}(Y) E_{i}^{*}(Z)+\cdots+E_{i-1}^{*}(Y) E_{i}^{*}(Z)\right) A_{i} \boldsymbol{v}+E_{i}^{*}(Y) A_{i} \boldsymbol{v} .
\end{aligned}
$$

Since the first term in the last expression is orthogonal to the last term, we have (i).

Since the right hand side of Theorem 10.1 does not depend on the choice of base subsets, for every $i \in\{0,1, \ldots, r(v)\}$ we have

$$
\left\|E_{i}^{*}(Y) A_{i} v\right\|^{2} \leq\left\|E_{i}^{*}(Z) A_{i} v\right\|^{2} \leq\left\langle g_{i}(t), g_{i}(t)\right\rangle\|v\|^{2},
$$

by (i). Thus the assertion follows from Theorem 10.1 (iii). 


\section{1. $Y$ with small width}

In this section we study irreducible modules of endpoint 0 when $w(Y)$ is small. If $w(Y)=0$, $Y$ consists of a single vertex $y$. Then $\mathcal{T}=\mathcal{T}(\{y\})$ is the Terwilliger algebra of [45]. If $v$ is a vector in $V$ with $\operatorname{supp}(v) \subset Y=\{y\}$, it is a scalar multiple of $\hat{y}$. Hence $\mathcal{T} v$ is the primary module and it is always thin. See [45, Lemma 3.6]. Hence we now consider the cases $w(Y)=1$ and $w(Y)=2$. For $M \in \mathcal{M}$, let $\tilde{M}=E_{0}^{*} M E_{0}^{*}$ and $\tilde{\mathcal{M}}=E_{0}^{*} \mathcal{M} E_{0}^{*}$.

\section{1. $w(Y)=1$}

Throughout this subsection assume that $w(Y)=1$.

Lemma 11.1 The following hold.

(i) $\tilde{\mathcal{M}}=\operatorname{Span}\left(\tilde{A}_{0}, \tilde{A}_{1}\right)=\operatorname{Span}(\tilde{I}, \tilde{J})$ is commutative.

(ii) $\theta_{D} \geq-k /(|Y|-1)$.

(iii) Let $\bar{v}$ be an eigenvector of $\tilde{A}$ in $E_{0}^{*} V$ associated with an eigenvalue $\eta$. Then

$$
\frac{\left\|E_{i} \boldsymbol{v}\right\|^{2}}{\|\boldsymbol{v}\|^{2}}=\frac{m_{i}\left(k+\eta \theta_{i}\right)}{k|X|}
$$

and one of the following holds.

(a) $E_{0} \boldsymbol{v} \neq \mathbf{0}, \eta=|Y|-1$, and $\boldsymbol{v}$ is a multiple of $\mathbf{1}_{0}$. Moreover, if $E_{i} \boldsymbol{v}=\mathbf{0}$ for some $i$, then $i=D, \theta_{D} \cdot \eta+k=0$ and $v$ is tight.

(b) $E_{0} \boldsymbol{v}=\mathbf{0}$ and $\eta=-1$. In particular, $\boldsymbol{v}$ is tight.

Proof: (i) is obvious.

Since $w(Y)=1$, the formula in Lemma 8.2 yields

$$
\frac{\left\|E_{i} \boldsymbol{v}\right\|^{2}}{\|\boldsymbol{v}\|^{2}}=\frac{1}{|X|}\left(q_{i}(0)+\eta q_{i}(1)\right)=\frac{m_{i}}{|X|}\left(1+\eta \frac{\theta_{i}}{k}\right)=\frac{m_{i}\left(k+\eta^{(1)}(\boldsymbol{v}) \theta_{i}\right)}{k|X|}
$$

as $\eta^{(0)}=1, \eta^{(1)}=\eta$.

Let $\boldsymbol{v}=\mathbf{1}_{0}$ be the characteristic vector of $Y$. Then $\eta=|Y|-1$, and

$$
0 \leq \frac{|X|\left\|E_{i} \boldsymbol{v}\right\|^{2}}{m_{i}\|\boldsymbol{v}\|^{2}}=1+\frac{(|Y|-1) \theta_{i}}{k}
$$

Hence we have (ii). Moreover $E_{i} \boldsymbol{v}=\mathbf{0}$ if and only if $i=D$ and $\theta_{D} \cdot(|Y|-1)+k=0$. Now the rest of the assertions follow from Definition 9.2.

Lemma 11.2 Let $v$ be an eigenvector of $\tilde{A}$ in $E_{0}^{*} V$ associated with an eigenvalue $\eta$. Then $\eta \neq 0$. Let $\rho(t)=\rho_{\boldsymbol{v}}(t)$ and let $\theta=-b_{0} / \eta$. Then $\theta=b_{0}=k$ or $\theta \leq \theta_{D}$. Moreover, the following hold.

(i) $\rho\left(\theta_{i}\right)=\frac{1}{|X|}\left(1+\frac{\eta}{k} \theta_{i}\right)=\frac{\left(\theta-\theta_{i}\right)}{\theta|X|} \geq 0$ for every $i \in\{0,1, \ldots, D\}$. 
(ii) $v_{i}(\theta) \neq 0$ for every $i \in\{0,1, \ldots, D\}$.

(iii) For $\ell \in\{0,1, \ldots, D\}$, let $g_{\ell}(t) \in \boldsymbol{R}[t]$ defined by the following.

$$
g_{\ell}(t)=\sum_{h=0}^{\ell} \frac{k_{\ell}}{k_{h}} \frac{v_{h}(\theta)}{v_{\ell}(\theta)} v_{h}(t) .
$$

Then for every $i, j \in\{0,1, \ldots, D\}$ the following hold.

$$
\sum_{h=0}^{D} g_{i}\left(\theta_{h}\right) g_{j}\left(\theta_{h}\right) \rho\left(\theta_{h}\right) m\left(\theta_{h}\right)=\delta_{i, j} k_{i} c_{i+1} \frac{v_{i+1}(\theta)}{\theta \cdot v_{i}(\theta)} .
$$

(iv) For every $\ell \in\{0,1, \ldots, D-1\}, g_{0}(t), g_{1}(t), \ldots, g_{D}(t)$ satisfy the following three term recurrence.

$$
\operatorname{tg}_{\ell}(t)=\beta_{\ell-1} g_{\ell-1}(t)+\alpha_{\ell} g_{\ell}(t)+\gamma_{\ell+1} g_{\ell+1}(t),
$$

where

$$
\begin{aligned}
\beta_{\ell-1} & =\frac{b_{\ell-1} c_{\ell+1} v_{\ell-1}(\theta) v_{\ell+1}(\theta)}{c_{\ell}\left(v_{\ell}(\theta)\right)^{2}}, \\
\alpha_{\ell} & =a_{\ell}+\frac{v_{\ell-1}(\theta)}{v_{\ell}(\theta)} b_{\ell-1}-\frac{v_{\ell}(\theta)}{v_{\ell+1}(\theta)} b_{\ell}, \\
\gamma_{\ell+1} & =c_{\ell+1} .
\end{aligned}
$$

Moreover, if $\theta<\theta_{D}$, then (32) holds for $\ell=D$ by setting $g_{D+1}(t)=v_{D+1}(t)$.

Proof: Since $\eta=|Y|-1$ or $-1, \eta \neq 0$. Now (i) follows from Lemma 11.1 (iii).

The proofs of (ii), (iii), and (iv) are very similar to those of Lemma 3.2 and therefore omitted. See [23, 48] and [5, Section 1.7].

Proposition 11.3 Let $\boldsymbol{v}$ be an eigenvector of $\tilde{A}$ in $E_{0}^{*} V$ such that $\tilde{A} \boldsymbol{v}=\eta \boldsymbol{v}$. Let $\boldsymbol{v}_{i}=E_{i}^{*} A_{i} \boldsymbol{v}$ for $i \in\{0,1, \ldots, D+1\}$. Then the following hold.

(i) If $\eta=-1$, then the module $\mathcal{T} v$ is always thin of dimension D. For $i \in\{0,1, \ldots, D-1\}$, $v_{i}=p_{i}(A) v$, where $p_{0}(t)=1, p_{1}(t), p_{2}(t), \ldots, p_{D}(t)$ is a system of orthogonal polynomials defined by

$$
t p_{i}(t)=b_{i} p_{i-1}(t)+\left(a_{i}-c_{i+1}+c_{i}\right) p_{i}(t)+c_{i+1} p_{i+1}(t) \quad(0 \leq i \leq D-1),
$$

where $p_{-1}(t)=0$. They satisfy $p_{i}\left(A_{1}\right)=A_{0}+A_{1}+\cdots+A_{i}$. Moreover, $\mathcal{T} \boldsymbol{v}=$ $\operatorname{Span}\left(\boldsymbol{v}_{0}, \boldsymbol{v}_{1}, \ldots, \boldsymbol{v}_{D-1}\right)$ and

$$
A \boldsymbol{v}_{i}=b_{i} \boldsymbol{v}_{i-1}+\left(a_{i}-c_{i+1}+c_{i}\right) \boldsymbol{v}_{i}+c_{i+1} \boldsymbol{v}_{i+1},
$$

for $i \in\{0,1, \ldots, D-1\}$ and $\boldsymbol{v}_{D}=\mathbf{0}$.

(ii) If $\eta=|Y|-1, \operatorname{dim} \mathcal{M} v$ is either $D$ or $D+1$. Let $\theta=-b_{0} / \eta$. For $i \in\{0,1, \ldots, D\}$, let $\beta_{i-1}, \alpha_{i}, \gamma_{i+1}$, and $g_{i}(t)$ be as in Lemma 11.2. Then the following hold.

(a) If $\operatorname{dim} \mathcal{M} v=D$, then $\theta=\theta_{D},|Y|=1-k / \theta_{D}$, and $\mathcal{T} v$ is always thin. Moreover, for $i \in\{0,1, \ldots, D-1\} \boldsymbol{v}_{i}=g_{i}(A) v, \mathcal{T} \boldsymbol{v}=\operatorname{Span}\left(\boldsymbol{v}_{0}, \boldsymbol{v}_{1}, \ldots, \boldsymbol{v}_{D-1}\right)$ and

$$
A \boldsymbol{v}_{i}=\beta_{i-1} \boldsymbol{v}_{i-1}+\alpha_{i} \boldsymbol{v}_{i}+\gamma_{i+1} \boldsymbol{v}_{i+1}
$$

with $\boldsymbol{v}_{-1}=\boldsymbol{v}_{D}=\mathbf{0}$. 
(b) If $\operatorname{dim} \mathcal{M} v=D+1$, then

$$
\begin{aligned}
& |Y|\left|\Gamma_{D}(Y)\right| \leq \frac{k_{D} v_{D+1}(\theta)}{\theta v_{D}(\theta)} \\
& \text { with } \theta=-k /(|Y|-1) \text { and } \mathcal{T} \boldsymbol{v} \text { is thin if and only if equality holds above. Moreover, } \\
& \text { for } i \in\{0,1, \ldots, D\} \boldsymbol{v}_{i}=g_{i}(A) \boldsymbol{v}, \mathcal{T} \boldsymbol{v}=\operatorname{Span}\left(\boldsymbol{v}_{0}, \boldsymbol{v}_{1}, \ldots, \boldsymbol{v}_{D}\right) \text { and } \\
& A \boldsymbol{v}_{i}=\beta_{i-1} \boldsymbol{v}_{i-1}+\alpha_{i} \boldsymbol{v}_{i}+\gamma_{i+1} \boldsymbol{v}_{i+1} \\
& \text { with } \boldsymbol{v}_{-1}=\boldsymbol{v}_{D+1}=\mathbf{0} \text {. }
\end{aligned}
$$

Proof: (i) In this case $\theta=b_{0}=k$. Hence $v_{i}(\theta)=k_{i}$ for every $i$. Moreover, $g_{i}(t)$ in Lemma 11.2 becomes $p_{i}(t)$ in Lemma 3.1 (ii). Hence the assertions follow from Lemma 3.1 (ii), Lemma 11.2 (iii), (iv), and Theorem 9.2 together with the fact in Lemma 11.1 (iii) (ii).

(ii) (a) follows similarly.

We need to consider the case when $\boldsymbol{v}=\mathbf{1}_{0}$ with $\operatorname{dim} \mathcal{M} \boldsymbol{v}=D+1$. Since $D$ is the diameter of $\Gamma, Y \subset \Gamma_{D}(x)$ for every $x \in \Gamma_{D}(Y)$. Hence

$$
\left\|E_{D}^{*} A_{D} \mathbf{1}_{0}\right\|^{2}=\left\||Y| \mathbf{1}_{D}\right\|^{2}=|Y|^{2}\left|\Gamma_{D}(Y)\right| .
$$

Since

$$
\left\langle g_{D}(t), g_{D}(t)\right\rangle_{\rho m}=k_{D} \frac{v_{D+1}(\theta)}{\theta \cdot v_{D}(\theta)}
$$

we have the assertion by Theorem 10.1 (ii) and (iii).

11.2. $w(Y)=2$

In this subsection we assume that $w(Y)=2$. This is the case when $Y=\Gamma_{1}(x)$ for some vertex $x$.

Lemma 11.4 Let $\boldsymbol{v} \in E_{0}^{*} V$ be a nonzero vector such that $E_{0} \boldsymbol{v}=\mathbf{0}$. Then

$$
\frac{\left\|E_{i} \boldsymbol{v}\right\|^{2}}{\|\boldsymbol{v}\|^{2}}=\frac{m_{i}\left(k-\theta_{i}\right)\left(\left(1+\eta^{(1)}(\boldsymbol{v})\right)\left(1+\theta_{i}\right)+b_{1}\right)}{k b_{1}|X|} .
$$

Proof: Set $\eta^{(i)}=\eta^{(i)}(\boldsymbol{v})$ and $\eta=\eta^{(1)}$. Since $E_{0} \boldsymbol{v}=\mathbf{0}$ and $w(Y)=2$,

$$
\eta^{(2)}\|\boldsymbol{v}\|^{2}={ }^{t} \boldsymbol{v} \tilde{A}_{2} \overline{\boldsymbol{v}}={ }^{t} \boldsymbol{v}\left(\tilde{J}-\tilde{A}_{1}-\tilde{A}_{0}\right) \overline{\boldsymbol{v}}=-(1+\eta)\|\boldsymbol{v}\|^{2} .
$$


Hence we have $\eta^{(2)}=-(1+\eta)$ and by (12) and (13),

$$
\begin{aligned}
q_{i}(0) & +\eta q_{i}(1)+\eta^{(2)} q_{i}(2) \\
= & m_{i}\left(1+\eta \frac{\theta_{i}}{k}-(1+\eta) \frac{\theta_{i}^{2}-a_{1} \theta_{i}-k}{k b_{1}}\right) \\
= & \frac{m_{i}}{k b_{1}}\left(k b_{1}+\eta b_{1} \theta_{i}-(1+\eta)\left(\theta_{i}^{2}-a_{1} \theta_{i}-k\right)\right) \\
= & \frac{m_{i}}{k b_{1}}\left(k b_{1}+\eta b_{1} \theta_{i}-\theta_{i}^{2}+a_{1} \theta_{i}+k-\eta \theta_{i}^{2}+a_{1} \eta \theta_{i}+k \eta\right) \\
= & \frac{m_{i}}{k b_{1}}\left(\left(k-\theta_{i}\right) b_{1}+\left(k-\theta_{i}\right) \theta_{i}+\left(k-\theta_{i}\right)+\eta\left(b_{1} \theta_{i}-\theta_{i}^{2}+a_{1} \theta_{i}+k\right)\right) \\
= & \frac{m_{i}\left(k-\theta_{i}\right)\left((1+\eta)\left(1+\theta_{i}\right)+b_{1}\right)}{k b_{1}} .
\end{aligned}
$$

Now the formula follows from Lemma 8.2.

Lemma 11.5 Suppose the induced subgraph on $Y$ is regular of valency $\kappa$ and size $m=|Y|$, i.e., $\tilde{A} \mathbf{1}_{0}=\kappa \mathbf{1}_{0}$. Let $\kappa=\eta_{1} \geq \eta_{2} \geq \cdots \geq \eta_{m}$ be the eigenvalues of $\tilde{A}$ on $E_{0}^{*} V$. Then the following hold.

(i) $\operatorname{tr}(\tilde{A})=\sum_{i=1}^{m} \eta_{i}=0$.

(ii) $\operatorname{tr}\left(\tilde{A}^{2}\right)=\sum_{i=1}^{m} \eta_{i}^{2}=m \eta_{1}$.

(iii) Suppose $w(Y)=2<D$, then

$$
-1-\frac{b_{1}}{1+\theta_{D}} \geq \eta_{2} \geq \cdots \geq \eta_{m} \geq-1-\frac{b_{1}}{1+\theta_{1}} .
$$

Proof: (i) and (ii) are well-known.

Let $\boldsymbol{v}$ be an eigenvector of $\tilde{A}$ associated with an eigenvalue $\eta=\eta_{i}$ with $i \geq 2$. Since $\mathbf{1}_{0}$ is an eigenvector for $\kappa=\eta_{1}$, we may assume that $\boldsymbol{v}$ is orthogonal to $\mathbf{1}_{0}$. Hence $E_{0} \boldsymbol{v}=\mathbf{0}$. By Lemma 11.4, we have

$$
0 \leq \frac{\left\|E_{i} \boldsymbol{v}\right\|^{2}}{\|\boldsymbol{v}\|^{2}}=\frac{m_{i}\left(k-\theta_{i}\right)\left((1+\eta)\left(1+\theta_{i}\right)+b_{1}\right)}{k b_{1}|X|} .
$$

Hence $(1+\eta)\left(1+\theta_{i}\right)+b_{1} \geq 0$. It is well-known that $\theta_{1}>-1>\theta_{D}$. (See for example [24, Section 13.2] or [31, Lemmas 2.3 and 2.6].) Hence we have

$$
-1-\frac{b_{1}}{1+\theta_{D}} \geq \eta \geq-1-\frac{b_{1}}{1+\theta_{1}} .
$$


Remark We remark that Lemma 11.5 (iii) is essentially proved in [31, 44].

Lemma 11.6 The following hold.

(i) $\tilde{\mathcal{M}}=\operatorname{Span}\left(\tilde{A}_{0}, \tilde{A}_{1}, \tilde{A}_{2}\right)=\operatorname{Span}\left(\tilde{I}, \tilde{A}_{1}, \tilde{J}\right) . \tilde{\mathcal{M}}$ is commutative if and only if the induced subgraph on $Y$ is regular.

(ii) Let $v$ be a common eigenvector of $\tilde{\mathcal{M}}$ in $E_{0}^{*} V$ such that $\tilde{A}_{1} v=\eta \boldsymbol{v}$. Then one of the following hold.

(a) $E_{0} \boldsymbol{v} \neq \mathbf{0}, \boldsymbol{v}$ is a multiple of $\mathbf{1}_{0}$, and the induced subgraph on $Y$ is regular of valency $\eta$. Moreover, if $\boldsymbol{v}$ satisfies the tight condition, then $E_{i} \boldsymbol{v}=E_{i+1} \boldsymbol{v}=\mathbf{0}$ for some index $i \in\{1, \ldots, D-1\}$.

(b) $E_{0} \boldsymbol{v}=\mathbf{0}$. Moreover, if $\boldsymbol{v}$ satisfies the tight condition, then either $E_{1} \boldsymbol{v}=\mathbf{0}$ or $E_{D} v=\mathbf{0}$.

Proof: (i) follows from the well-known fact that the adjacency matrix of a graph commutes with the all-one's matrix if and only if the graph is regular.

(ii) Let $\rho(t)=\rho_{\boldsymbol{v}}(t)$. Then the degree of $\rho(t)$ is at most 2 and $\rho\left(\theta_{h}\right) \geq 0$ for every $h \in\{0,1, \ldots, D\}$. Hence if $\rho\left(\theta_{i}\right)=\rho\left(\theta_{j}\right)=0$ for some $i<j$ with $i, j \in\{0,1, \ldots, D\}$, then $\rho(t)$ is quadratic and either $j=i+1$ or $i=0$ and $j=D$. If $\rho\left(\theta_{0}\right)=0$, then we have case (b). Now we have the assertions by Theorem 9.2.

Definition 11.1 For each element $z \in R \backslash\{-1\}$ set

$$
\tilde{z}=-1-\frac{b_{1}}{1+z}
$$

Let $\left\{p_{0}(t), p_{1}(t), \ldots, p_{D}(t)\right\}$ be the system of polynomials in Lemma 3.1. Let $\theta$ be a real number satisfying $\theta \geq \theta_{1}$ or $\theta \leq \theta_{D}$. For $\ell \in\{0,1, \ldots, D\}$, let $g_{\ell}(t)$ be the polynomial defined in Lemma 3.2 .

Proposition 11.7 Suppose the induced subgraph on $Y$ is regular of valency $\kappa$. Let $\boldsymbol{v}$ be an eigenvector of $\tilde{A}_{1}$ in $E_{0}^{*} V$ such that $\tilde{A} \boldsymbol{v}=\eta \boldsymbol{v}$. Assume that $E_{0} \boldsymbol{v}=\mathbf{0}$. Then the following hold.

(i) The following hold.

$$
\begin{aligned}
\rho_{\boldsymbol{v}}\left(\theta_{i}\right) m\left(\theta_{i}\right) & =\frac{\left\|E_{i} \boldsymbol{v}\right\|^{2}}{\|\boldsymbol{v}\|^{2}}=\frac{m_{i}\left(k-\theta_{i}\right)\left((1+\eta)\left(1+\theta_{i}\right)+b_{1}\right)}{k b_{1}|X|} \\
& = \begin{cases}\frac{m_{i}\left(k-\theta_{i}\right)\left(\tilde{\eta}-\theta_{i}\right)}{k(1+\tilde{\eta})|X|} & \text { for } \eta \neq-1, \\
\frac{m_{i}\left(k-\theta_{i}\right)}{k|X|} & \text { for } \eta=-1 .\end{cases}
\end{aligned}
$$

(ii) $v$ is tight with respect to $Y$, i.e., $r(v)=D-2$, if and only if $\eta=\tilde{\theta}_{1}$, or $\tilde{\theta}_{D}$. In this case $\mathcal{T} v$ is a thin irreducible module of dimension $D-1$. Moreover, if we set $\theta=\tilde{\eta}$ to 
define polynomials $g_{i}(t)$ 's, then $\boldsymbol{v}_{i}=E_{i}^{*} A_{i} v=g_{i}(A) v$ for $i \in\{0,1, \ldots, D-1\}$ with $\boldsymbol{v}_{D-1}=\mathbf{0}$. Moreover, for $i \in\{0,1, \ldots, D-2\}$,

$$
A \boldsymbol{v}_{i}=\beta_{i-1} \boldsymbol{v}_{i-1}+\alpha_{i} \boldsymbol{v}_{i}+\gamma_{i+1} \boldsymbol{v}_{i+1}
$$

Proof: (i) This follows from the equation

$$
(1+\tilde{\eta})(1+\eta)=-b_{1}
$$

(ii) $r(\boldsymbol{v})+1=\operatorname{dim} \mathcal{M} \boldsymbol{v}=D-1$ if and only if $\rho_{\boldsymbol{v}}\left(\theta_{i}\right)=0$ for some $i \in\{1,2, \ldots, D\}$. It follows from Lemma 11.5 (iii) together with (i) above that it is equivalent to $\eta \in\left\{\tilde{\theta}_{1}, \tilde{\theta}_{D}\right\}$. In this case $\mathcal{T} v$ is thin by Theorem 9.2. Since $\eta \neq-1,\left\{g_{0}(t), g_{1}(t), \ldots, g_{D-2}(t)\right\}$ satisfies

$$
\left\langle g_{i}(t), g_{j}(t)\right\rangle_{\omega}=\delta_{i, j} \frac{p_{i+1}(\tilde{\eta}) c_{i+1}}{p_{i}(\tilde{\eta})(\tilde{\eta}+1)} \frac{k_{i} b_{i}}{k}
$$

by Lemma 3.2 (iv) and (i) above.

Since the leading coefficient of $g_{i}(t)$ coincides with that of $v_{i}(t)$, we have the assertions by Lemma 3.2 and Theorem 10.1. The last three term recursion is obtained from Lemma 3.2 (iii).

Proposition 11.8 Suppose the induced subgraph on $Y$ is regular of valency $\kappa$. Let $\boldsymbol{v}$ be an eigenvector of $\tilde{A}_{1}$ in $E_{0}^{*} V$ such that $\tilde{A} \boldsymbol{v}=\eta \boldsymbol{v}$. Assume that $E_{0} \boldsymbol{v}=\mathbf{0}$. Let $\rho(t)=\rho_{\boldsymbol{v}}(t)$. If $\operatorname{dim} \mathcal{M} v=D$, then the following hold.

(i) $\Theta(v)=\left\{\theta_{1}, \theta_{2}, \ldots, \theta_{D}\right\}$ and $\tilde{\eta}>\theta_{1}$ or $\tilde{\eta}<\theta_{D}$.

(ii) If $\eta \neq-1$, then the system of polynomials $\left\{g_{0}(t), g_{1}(t), \ldots, g_{D-1}(t)\right\}$ is the system of orthogonal polynomials associated with the weight function $\omega=\rho \cdot m$ on $\Theta(v)$ such that the leading coefficient of $g_{\ell}(t)$ coincides with that of $v_{\ell}(t)$ and $p_{\ell}(t)$. Moreover, the following hold.

(a) $\left\langle g_{i}(t), g_{j}(t)\right\rangle_{\omega}=\delta_{i, j} \frac{p_{i+1}(\tilde{\eta}) c_{i+1} k_{i} b_{i}}{p_{i}(\tilde{\eta})(\tilde{\eta}+1) k}$.

(b) $\left\|E_{i}^{*} A_{i} v\right\|^{2} \leq\left\langle g_{i}(t), g_{i}(t)\right\rangle_{\omega}\|\boldsymbol{v}\|^{2}$ and the equality holds if and only if $\mathcal{T} v$ is irreducible and thin.

(c) If $\mathcal{T} \boldsymbol{v}$ is an irreducible thin module, then $\boldsymbol{v}_{i}=E_{i}^{*} A_{i} \boldsymbol{v}=g_{i}(A) \boldsymbol{v}$ for $i \in\{0,1, \ldots$, $D\}$ with $\boldsymbol{v}_{D}=\mathbf{0}$. Moreover, for $i \in\{0,1, \ldots, D-1\}$,

$$
A \boldsymbol{v}_{i}=\beta_{i-1} \boldsymbol{v}_{i-1}+\alpha_{i} \boldsymbol{v}_{i}+\gamma_{i+1} \boldsymbol{v}_{i+1} \text {. }
$$

(iii) If $\eta=-1$, then the system of polynomials $\left\{p_{0}(t), p_{1}(t), \ldots, p_{D-1}(t)\right\}$ is the system of orthogonal polynomials associated with the weight function $\omega=\rho \cdot m$ on $\Theta(v)$ such that the leading coefficient of $p_{\ell}(t)$ coincides with that of $v_{\ell}(t)$. Moreover, the following hold.

(a) $\left\langle p_{i}(t), p_{j}(t)\right\rangle_{\omega}=\delta_{i, j} \frac{k_{i} b_{i}}{k}$.

(b) $\left\|E_{i}^{*} A_{i} v\right\|^{2} \leq\left\langle p_{i}(t), p_{i}(t)\right\rangle_{\omega}\|v\|^{2}$ and the equality holds if and only if $\mathcal{T} v$ is irreducible and thin. 
(c) If $\mathcal{T} v$ is an irreducible thin module, then $\boldsymbol{v}_{i}=E_{i}^{*} A_{i} v=p_{i}(A) v$ for $i \in\{0,1, \ldots$, $D\}$ with $\boldsymbol{v}_{D}=\mathbf{0}$. Moreover, for $i \in\{0,1, \ldots, D-1\}$,

$$
A \boldsymbol{v}_{i}=b_{i} \boldsymbol{v}_{i-1}+\left(a_{i}+c_{i}-c_{i+1}\right) \boldsymbol{v}_{i}+c_{i+1} \boldsymbol{v}_{i+1} \text {. }
$$

Proof: By Proposition 11.7 (i),

$$
\rho_{\boldsymbol{v}}\left(\theta_{i}\right) m\left(\theta_{i}\right)= \begin{cases}\frac{m_{i}\left(k-\theta_{i}\right)\left(\tilde{\eta}-\theta_{i}\right)}{k(1+\tilde{\eta})|X|} & \text { for } \eta \neq-1, \\ \frac{m_{i}\left(k-\theta_{i}\right)}{k|X|} & \text { for } \eta=-1 .\end{cases}
$$

Hence if $\eta \neq-1$, then $\left\{g_{0}(t), g_{1}(t), \ldots, g_{D-1}(t)\right\}$ is a system of orthogonal polynomials with respect to the weight function $\omega=\rho \cdot m$, and if $\eta=-1$, then $\left\{p_{0}(t), p_{1}(t), \ldots\right.$, $\left.p_{D-1}(t)\right\}$ is the one which plays the same role. Now the assertions are direct consequences of Lemma 3.2 and Theorem 10.1.

\section{Acknowledgments}

The author would like to give special thanks to Professor Paul Terwilliger for sending his preprints and giving valuable suggestions, comments and encouragement. All motivations and ideas were given through his lectures given personally to the author.

\section{References}

1. E. Bannai and T. Ito, Algebraic Combinatorics I, Benjamin/Cummings, California, 1984.

2. A.E. Brouwer, A.M. Cohen, and A. Neumaier, Distance-Regular Graphs, Springer Verlag, Berlin, Heidelberg, 1989.

3. J.S. Caughman IV, "Spectra of bipartite $P$ - and $Q$-polynomial association schemes," Graphs Combin. 14 (1998), 321-343.

4. J.S. Caughman IV, "The Terwilliger algebras of bipartite $P$ - and $Q$-polynomial association schemes," Discrete Math. 196 (1999), 65-95.

5. T.S. Chihara, An Introduction to Orthogonal Polynomials, Mathematics and its Applications, 13 (1978), Gordon and Breach Science Publishers.

6. B. Collins, "The girth of a thin distance-regular graph," Graphs Combin. 13 (1997), 21-30.

7. B. Collins, "The Terwilliger algebra of an almost-bipartite distance-regular graph and its antipodal 2-cover," Discrete Math. 216 (2000), 35-69.

8. B. Curtin, "Bipartite distance-regular graphs I," Graphs Combin. 15 (1999), 143-158.

9. B. Curtin, "Bipartite distance-regular graphs II," Graphs Combin. 15 (1999), 377-391.

10. B. Curtin, "2-homogeneous bipartite distance-regular graphs," Discrete Math. 187 (1998), 39-70.

11. B. Curtin, "Distance-regular graphs with support a spin model are thin," in 16th British Combinatorial Conference (London, 1997), Discrete Math. 1999, vol. 197/198, pp. 205-216.

12. B. Curtin, "Almost 2-homogeneous bipartite distance-regular graphs," Europ. J. Combin. 21 (2000), 865876 .

13. B. Curtin and K. Nomura, "Distance-regular graphs related to the quantum enveloping algebra of $s l(2)$," $J$. Alg. Combin. 12 (2000), 25-36. 
14. B. Curtin, "The local structure of a bipartite distance-regular graph," Europ. J. Combin. 20 (1999), 739758.

15. P. Delsarte, "An algebraic approach to the association schemes of coding theory," Phillips Research Reports Suppl. 10 (1973).

16. G. Dickie, “Twice $Q$-polynomial distance-regular graphs are thin,” Europ. J. Combin. 16 (1995), 555-560.

17. G. Dickie and P. Terwilliger, "A note on thin $P$-polynomial and dual-thin $Q$-polynomial symmetric association schemes," J. Alg. Combin. 7 (1998), 5-15.

18. E. Egge, “A generalization of the Terwilliger algebra," J. Algebra 233 (2000), 213-252.

19. E. Egge, "The generalized Terwilliger algebra and its finite dimensional modules when $d=2$," Journal of Algebra 250 (2002), 178-216.

20. M.A. Fiol and E. Garriga, "On the algebraic theory of pseudo-distance-regularity around a set," Linear Algebra Appl. 298 (1999), 115-141.

21. M.A. Fiol and E. Garriga, "An algebraic characterization of completely regular codes in distance-regular graphs," SIAM J. Discrete Math. 15 (2001/02), 1-13.

22. J.T. Go, "The Terwilliger algebra of the Hypercube," Europ. J. Combin. 23 (2002), 399-430.

23. J.T. Go and P. Terwilliger, "Tight distance-regular graphs and the subconstituent algebra," Europ. J. Combin. 23 (2002), 793-816.

24. C.D. Godsil, Algebraic Combinatorics, Chapman and Hall, Inc., New York, 1993.

25. M. Giudice and C.E. Praeger, "Completely transitive codes in Hamming graphs," European J. Combin. 20 (1999), 647-661.

26. S.A. Hobart and T. Ito, "The structure of nonthin irreducible $T$-modules: Ladder bases and classical parameters," J. Alg. Combin. 7 (1998), 53-75.

27. A. Jurišić and J. Koolen, "A local approach to 1-homogeneous graphs," Designs, Codes, and Cryptography 21 (2000), 127-147.

28. A. Jurišić and J. Koolen, "Nonexistence of some antipodal distance-regular graphs of diameter four," Europ. J. Combin. 21 (2000), 1039-1046.

29. A. Jurišić and J. Koolen, "1-homogeneous graphs with cocktail party $\mu$-graphs,” J. Alg. Combin. 18 (2003), 79-98.

30. A. Jurišić and J. Koolen, "Krein parameters and antipodal tight graphs with diameter 3 and 4," Discrete Math. 244 (2002), 181-202.

31. A. Jurišić, J. Koolen, and P. Terwilliger, “Tight distance-regular graphs,” J. Alg. Combin. 12 (2000), 163-197.

32. M. MacLean, "An inequality involving two eigenvalues of a bipartite distance-regular graph," Discrete Math. 225 (2000), 193-216.

33. M. MacLean, “Taut distance-regular graphs of odd diameter,” J. Alg. Combin. 17 (2003), 125-148.

34. W.J. Martin, "Completely regular designs," J. Combin. Des. 6 (1998), 261-273.

35. A. Neumaier, "Completely regular codes," Discrete Math. 106/107 (1992), 353-360.

36. A.A. Pascasio, "Tight graphs and their primitive idempotents," J. Alg. Combin. 10 (1999), 47-59.

37. A.A. Pascasio, "Tight distance-regular graphs and $Q$-polynomial property," Graphs Combin. 17 (2001), 149169.

38. A.A. Pascasio, "An inequality on the cosine of a tight distance-regular graph," Linear Algebra Appl. 325 (2001), 147-159.

39. A.A. Pascasio, "An inequality in character algebra," Discrete Math. 264 (2003), 201-210.

40. G. Szegö, Orthogonal Polynomials, American Mathematical Society, Providence, Rhode Island, 1939.

41. H. Suzuki, "Distance semi-regular graphs," Algebraic Colloquium 2 (1995), 315-328.

42. H. Suzuki, "An introduction to distance-regular graphs," Lecture Note, in Three Lectures in Algebra, Sophia University Lecture Note Series No. 41 (1999), pp. 57-132.

43. K. Tanabe, "The irreducible modules of the Terwilliger algebras of Doob schemes," J. Alg. Combin. 6 (1997), 173-195.

44. P. Terwilliger, “A new feasibility condition for distance-regular graphs," Discrete Math. 61 (1986), 311-315.

45. P. Terwilliger, "The subconstituent algebra of an association scheme, (Part I)," J. Alg. Combin. 1 (1992), $363-388$.

46. P. Terwilliger, "The subconstituent algebra of an association scheme, (Part II)," J. Alg. Combin. 2 (1993), 73-103. 
47. P. Terwilliger, "The subconstituent algebra of an association scheme, (Part III)," J. Alg. Combin. 2 (1993), $177-210$.

48. P. Terwilliger, "The subconstituent algebra of a distance-regular graph; thin modules with endpoint one," Linear Algebra Appl. 356 (2002), 157-187.

49. P. Terwilliger, "An inequality involving the local eigenvalues of a distance-regular graph,” J. Alg. Combin. 19 (2004), 143-172.

50. M. Tomiyama and N. Yamazaki, "The subconstituent algebra of a strongly regular graph," Kyushu J. Math. 48 (1998), 323-334. 